\title{
Obesity-induced excess of 17-hydroxyprogesterone promotes hyperglycemia through activation of glucocorticoid receptor
}

\author{
Yan Lu, ${ }^{1}$ E Wang, ${ }^{1}$ Ying Chen, ${ }^{1}$ Bing Zhou, ${ }^{1}$ Jiejie Zhao, ${ }^{1}$ Liping Xiang, ${ }^{1}$ Yiling Qian, ${ }^{1}$ Jingjing Jiang, ${ }^{1}$ Lin Zhao, ${ }^{1}$ Xuelian Xiong, \\ Zhiqiang Lu, ${ }^{1}$ Duojiao Wu, ${ }^{2}$ Bin Liu, ${ }^{1,3}$ Jing Yan, ${ }^{4}$ Rong Zhang, ${ }^{4,5}$ Huijie Zhang, ${ }^{6}$ Cheng Hu, ${ }^{4,5,7}$ and Xiaoying Li ${ }^{1}$ \\ ${ }^{1}$ Key Laboratory of Metabolism and Molecular Medicine, Ministry of Education and Department of Endocrinology and Metabolism, and ${ }^{2}$ Institute of Clinical Science, Shanghai Institute of Clinical \\ Bioinformatics, Zhongshan Hospital, Fudan University, Shanghai, China. 3liangsu Key Laboratory of Marine Pharmaceutical Compound Screening, College of Pharmacy, Jiangsu Ocean University, \\ Lianyungang, China. ${ }^{4}$ Department of Endocrinology and Metabolism, Shanghai Jiao Tong University Affiliated Sixth People's Hospital, and ${ }^{5}$ Shanghai Diabetes Institute, Shanghai Key Laboratory of Diabetes \\ Mellitus, Shanghai Clinical Center for Diabetes, Shanghai, China. ${ }^{6}$ Department of Endocrinology and Metabolism, Nanfang Hospital, Southern Medical University, Guangzhou, China. Institute for Metabolic \\ Disease, Fengxian Central Hospital, Southern Medical University, Shanghai, China.
}

\begin{abstract}
Type 2 diabetes mellitus (T2DM) has become an expanding global public health problem. Although the glucocorticoid receptor (GR) is an important regulator of glucose metabolism, the relationship between circulating glucocorticoids (GCs) and the features of T2DM remains controversial. Here, we show that 17-hydroxyprogesterone (17-OHP), an intermediate steroid in the biosynthetic pathway that converts cholesterol to cortisol, binds to and stimulates the transcriptional activity of GR. Hepatic 17-OHP concentrations are increased in diabetic mice and patients due to aberrantly increased expression of Cyp17A1. Systemic administration of 17-0HP or overexpression of Cyp17A1 in the livers of lean mice promoted the pathogenesis of hyperglycemia and insulin resistance, whereas knockdown of Cyp17A1 abrogated metabolic disorders in obese mice. Therefore, our results identify a Cyp17A1/17-OHP/CR-dependent pathway in the liver that mediates obesity-induced hyperglycemia, suggesting that selectively targeting hepatic Cyp17A1 may provide a therapeutic avenue for treating T2DM.
\end{abstract}

\section{Introduction}

In mammals, the liver is a master organ in the regulation of glucose and lipid metabolism in response to nutritional and hormonal stimuli (1). During the fasting state, hepatic gluconeogenesis is increased to maintain blood glucose levels within a narrow range, while ketone bodies, produced as a result of fatty acid oxidation, provide metabolic energy for other organs (2). In contrast, after a meal, circulating blood insulin and nutrients inhibit gluconeogenesis, activate glycogen synthesis, and stimulate de novo lipogenesis (2). In obesity, gluconeogenesis, hepatic glucose production, and de novo lipogenesis are enhanced due to nutritional excess and selective insulin resistance, leading to the development of type 2 diabetes mellitus (T2DM) $(3,4)$. Although enormous progress has been made in our understanding of the molecular mechanisms of T2DM, the major causative factors that lead to insulin resistance and hyperglycemia remain to be determined.

Glucocorticoids (GCs), steroid hormones derived from the adrenal gland, play an integral role in many physiological systems,

Authorship note: YL, EW, and YC contributed equally to this work. Conflict of interest: The authors have declared that no conflict of interest exists. Copyright: (c) 2020, American Society for Clinical Investigation. Submitted: October 23, 2019; Accepted: April 8, 2020; Published: June 8, 2020. Reference information: / Clin Invest. 2020;130(7):3791-3804. https://doi.org/10.1172/JCl134485. including growth, reproduction, inflammation, and metabolism (5). However, an excess of GCs has been implicated in the pathogenesis of metabolic disorders. Patients with Cushing syndrome, a condition characterized by elevated endogenous cortisol, usually exhibit hyperglycemia, insulin resistance, and liver steatosis (6-8). Furthermore, long-term use of GC synthetic analogs, such as dexamethasone (Dex) and hydrocortisone, has been linked to deleterious metabolic effects $(9,10)$. At the cellular level, the effects of GCs are mediated by the glucocorticoid receptor (GR), a member of the nuclear receptor superfamily $(11,12)$. GR resides in the cytoplasm in an unbound form and translocates into the nucleus upon binding to GCs; it also serves as a transcription factor for enhancing the expression of GC-responsive target genes $(11,12)$. In the liver, the GR is believed to be a critical transcriptional checkpoint for hyperglycemia, insulin resistance, gluconeogenesis, and hepatic steatosis (13), which are mediated through direct and indirect mechanisms. GR has been shown to upregulate gluconeogenic enzymes (e.g., PEPCK and G6Pase) through direct recruitment to their promoter regions $(14,15)$ and through activation of other important gluconeogenic transcription factors (e.g., KLF9 and CREBH) $(16,17)$. In contrast, inactivation of the GR gene in the liver markedly ameliorates hyperglycemia and hepatosteatosis in obese mice (18-21).

However, in contrast to the well-defined downstream functions and molecular pathways of GR, the factors contributing to the activation of GR in the liver remain uncertain. In particular, the relationship between the features of obesity or metabolic syndrome 
and cortisol concentrations in humans by multiple measurements, including morning and evening salivary cortisol as well as morning plasma cortisol, is variable (22). While some studies demonstrated a positive association between circulating cortisol levels and metabolic parameters, other studies found no relationship or even a negative association (22-27). Therefore, population-based observational reports strongly suggest that activation of GR in the liver of obese subjects may not be attributed to circulating GCs. Thus, identification of the molecules that modulate GR activity directly in the liver might provide a unique opportunity for understanding the molecular mechanisms that contribute to T2DM and also provide an option for therapeutic intervention.

The synthesis of biologically active steroid hormones from cholesterol generates and involves multiple intermediate products, including pregnenolone, 17-hydroxypregnenolone, progesterone, 17-hydroxyprogesterone (17-OHP), etc. Intriguingly, emerging evidence has shown that some derivatives of cholesterol, such as 25-hydroxycholesterol, 27-hydroxycholesterol, and 22(R)hydroxycholesterol, can function as endogenous ligands for several nuclear receptors, including liver X receptor (LXR), estrogen receptor $\alpha(\mathrm{ER} \alpha)$, and retinoic acid receptor-related orphan receptors (RORs) (28-30). We therefore tested to determine whether any of these steroid hormone precursors could be agonist ligands of GR and contribute to the development of metabolic disorders.

\section{Results}

17-OHP activates GR in hepatocytes. To test our hypothesis, human HepG2 cells were transfected with a luciferase reporter harboring 3 GR-responsive elements (GREs) and a GR expression plasmid. Transfected cells were deprived of FBS for 12 hours and subsequently exposed to increasing amounts of steroid hormone precursors. Dex, a synthetic GC analog, was employed as a positive control. Notably, only 17-OHP elicited a dramatic induction in GR transactivation activity (Figure 1A). However, deletion of the DNA-binding domain or ligand-binding domain of GR abolished the response to 17-OHP (Figure 1B). The ability of GR to respond to $17-\mathrm{OHP}$ is very specific. No transactivation was seen in the other nuclear receptors tested (Figure 1C). The selectivity of these nuclear receptors was confirmed via luciferase reporter assays using their specific ligands (Supplemental Figure 1, A-D; supplemental material available online with this article; https:/doi. org/10.1172/JCI134485DS1). Furthermore, a luciferase reporter containing the PEPCK gene promoter, which contains a functional GR response element $(14,31)$, was also stimulated by $17-\mathrm{OHP}$ (Figure 1D). Endogenous expression levels of direct GR target genes in mouse primary hepatocytes (MPHs), such as PEPCK and TAT (31), were upregulated by 17-OHP (Figure 1E). This induction was suppressed by RU486, an antagonist of both the GR and the progesterone receptor (PR) (Figure 1F). Due to the absent expression of PR in the liver, as shown by a previous report (32) and our Western blots (Supplemental Figure 1E), the suppressive role of RU486 suggested that induction of PEPCK and TAT by 17-OHP in MPHs depends on the GR instead of PR. Furthermore, expression levels of downstream target genes of other nuclear receptors, including SHP (common target gene of ER $\alpha$ and FXR) (33), Abca1 (target gene of LXR) (34), Cyp4A14 (target gene of PPAR $\alpha$ ) (35), and CD36 (common target gene of PPAR $\gamma$ and PXR) (36), were not affected by 17-OHP treatment (Supplemental Figure 1, F-I), further supporting the specificity of 17-OHP in activating the GR. In addition, in response to 17-OHP treatment, endogenous GR translocated into the nucleus (Figure 1G) and ChIP assays showed an increased recruitment of GR to the promoter regions of both PEP$\mathrm{CK}$ and TAT in MPHs (Figure 1H). To assess whether 17-OHP was a direct GR agonist, we used a cell-free coactivator recruitment assay. In this setting, interactions between the human recombinant GR ligand-binding domain and a fluorescein-labeled coactivator peptide were measured by fluorescence resonance energy transfer (FRET). We found 17-OHP activated the ligand-binding domain of $\mathrm{GR}$ with an $\mathrm{EC}_{50}$ of $7.14 \mu \mathrm{M}$ (Figure 1I). Therefore, our data suggest that 17-OHP can act as an endogenous GR ligand.

Hepatic 17-OHP concentration is elevated in T2DM. We next determined whether hepatic 17-OHP concentration is altered in mouse models of obesity. Liver tissues of C57BL/6 mice fed a high-fat diet (HFD) or normal chow diet (NCD) for 12 weeks were collected, homogenized, and measured by ELISAs. We found hepatic 17-OHP concentrations were markedly elevated in HFD-induced obese mice compared with NCD-fed lean mice (Figure 2A). Hepatic 17-OHP levels were also elevated in leptin receptor-deficient $d b / d b$ mice and leptin-deficient $o b / o b$ mice, 2 common models of severe obesity (Figure 2, B and C). Interestingly, plasma 17-OHP levels were also elevated in obese mice (Figure 2, D-F). Furthermore, 17-OHP concentrations in the liver correlated with circulating 17-OHP levels (Supplemental Figure 2, A-C), suggesting that elevated plasma 17-OHP concentrations in obesity, at least in part, are derived from the liver tissues.

To investigate whether circulating 17-OHP levels are also increased in obese human populations, morning plasma samples were collected from a cohort of age-matched healthy male subjects (BMI: $22.47 \pm 2.10 \mathrm{~kg} / \mathrm{m}^{2}$; HbA1c: $5.24 \% \pm 0.28 \%$ ) and patients with T2DM (BMI: $30.92 \pm 2.61 \mathrm{~kg} / \mathrm{m}^{2}$; HbA1c: $8.87 \% \pm 1.90 \%$ ). Steroid hormones were analyzed by liquid chromatographytandem mass spectrometry (LC-MS/MS) (Supplemental Table 1). Our results revealed an increase in plasma 17-OHP levels in T2DM patients compared with healthy subjects (Supplemental Table 1). We also confirmed that plasma levels of dehydroepiandrosterone (DHEAS) were reduced in diabetic patients (Supplemental Table 1), which has been reported previously (37-39).

To further explore the association between circulating 17-OHP levels and glucose metabolism, a total of 203 subjects of Han Chinese origin were recruited (40-42), and their circulating 17-OHP levels were measured by ELISA. Of note, plasma 17-OHP levels were higher in participants with diabetes or prediabetes compared with nondiabetic participants (both $P<0.05$, Table 1). We further divided the participants into 4 groups according to quartiles of plasma 17-OHP levels (Table 2). Participants in the higher quartiles of plasma 17-OHP levels had higher levels of fasting plasma glucose, 2-hour load plasma glucose, HbA1c, triglycerides (TGs), and free fatty acids (FFAs) ( $P$ $<0.001$ for trend). Pearson's correlation analysis showed that fasting plasma glucose, along with 2-hour postload plasma glucose, HbA1c, plasma TGs, FFAs, uric acid, systolic blood pressure, and diastolic blood pressure, was significantly positively correlated with plasma 17-OHP levels (Figure 2, G-N). No significant correlations were found between plasma 17-OHP levels and 
A

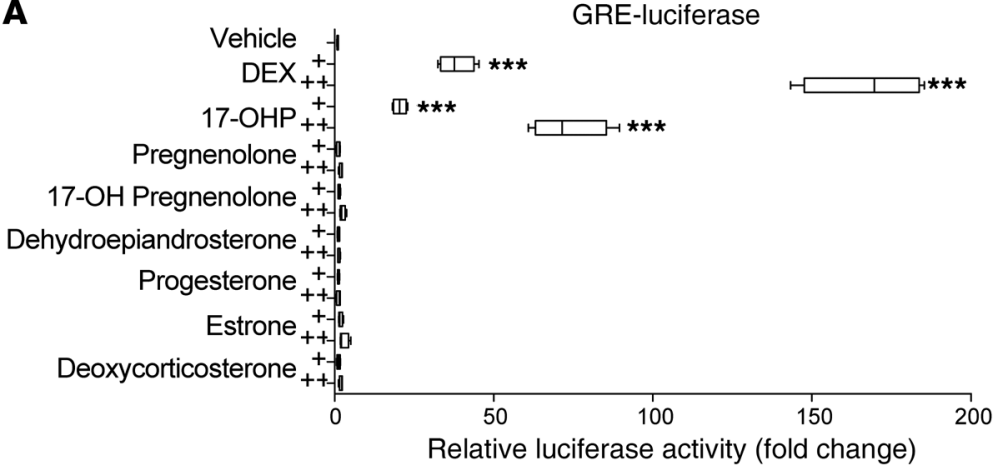

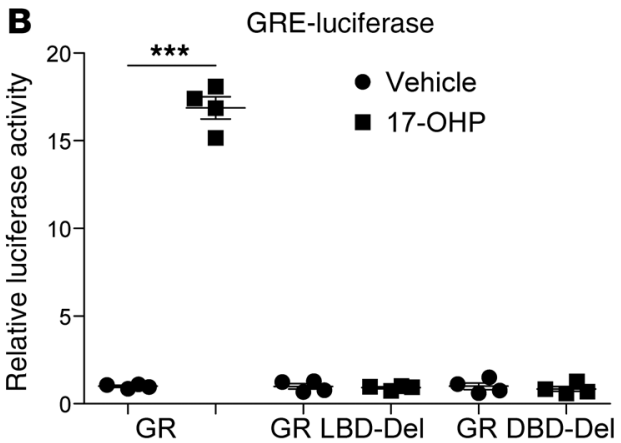
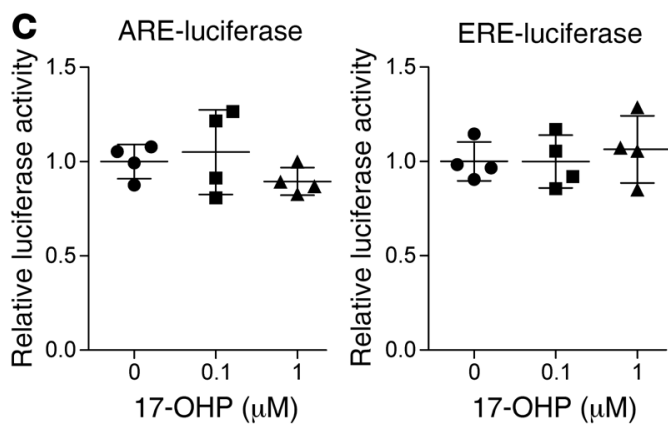
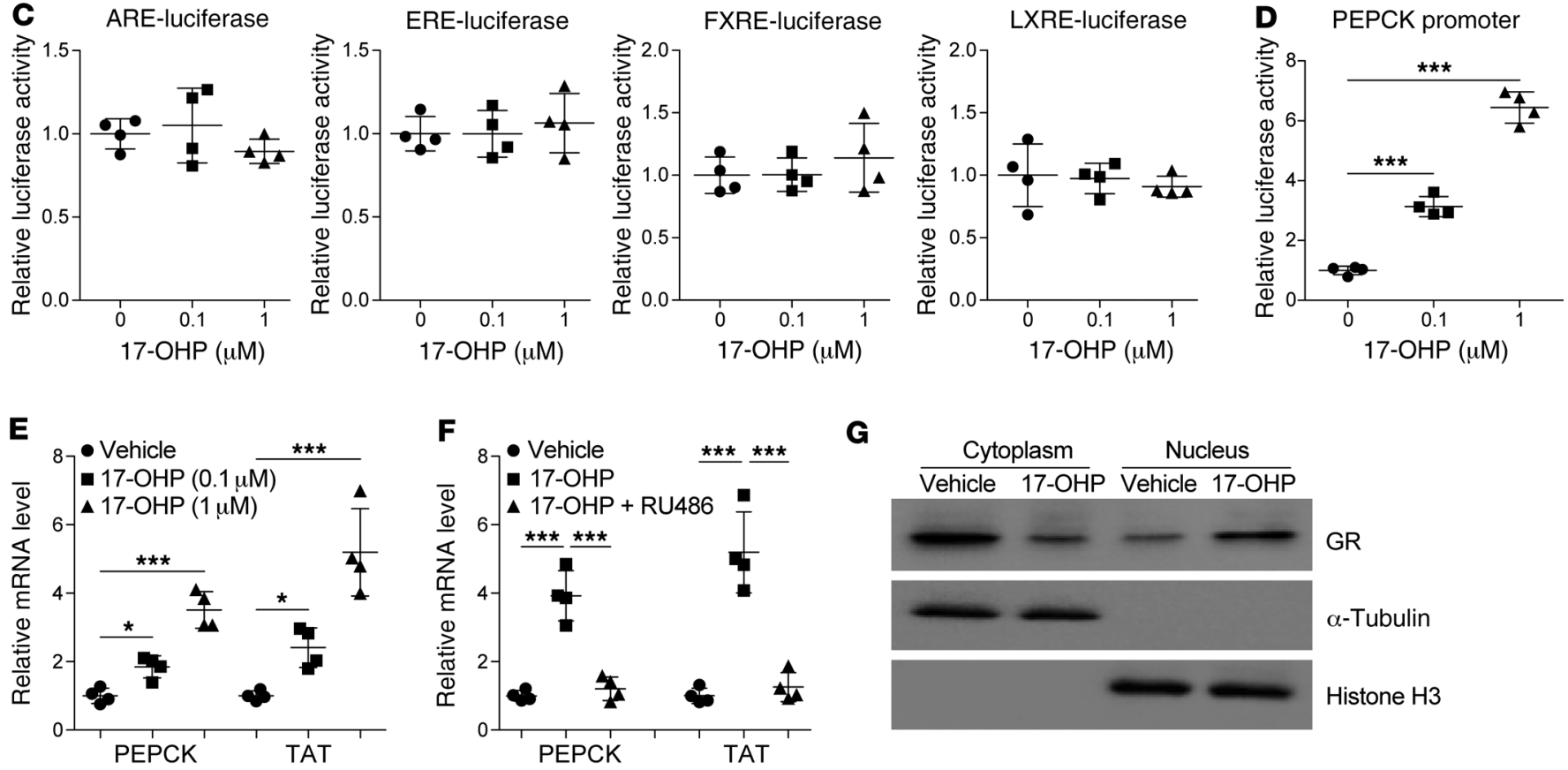

H

PEPCK promoter TAT promoter GAPDH promoter

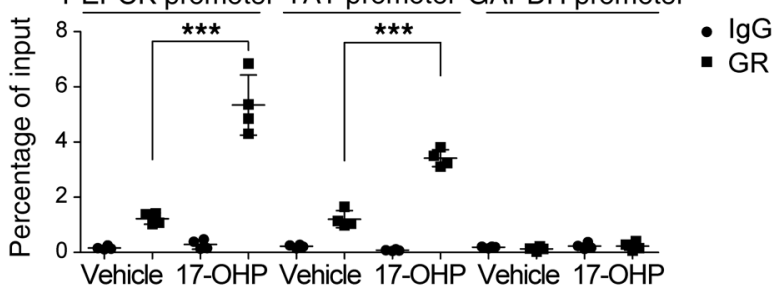

I

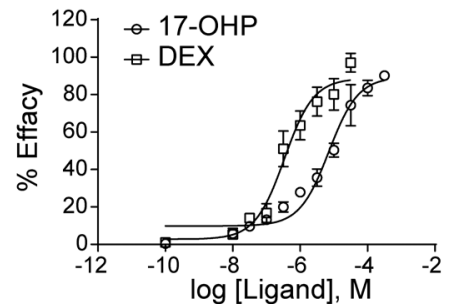

Figure 1. 17-OHP induces GR transcriptional activity. (A) Activation of GR by increasing doses of steroid hormone intermediates. HepG2 cells were transfected with a GRE-luciferase reporter and GR expression plasmid. Cells were deprived of FBS for 12 hours and treated with steroid hormone precursors for another 24 hours. Vehicle, ethanol; Dex (+, $10 \mathrm{nM} ;++, 100 \mathrm{nM}) ; 17-\mathrm{OHP}(+, 0.1 \mu \mathrm{M} ;++, 1 \mu \mathrm{M})$; other intermediates (+, $0.1 \mu \mathrm{M} ;++, 1 \mu \mathrm{M})$. (B) Luciferase assays for GRE activity in HepG2 cells treated with or without 17-OHP (0.1 $\mu \mathrm{M})$ for 24 hours. Cells were transfected with a GRE-luciferase reporter and intact GR or 2 truncated GR expression plasmids, as indicated. The first and second columns represent WT GR expression plasmid. The third and fourth columns represent LBD-Del GR expression plasmid. The fifth and sixth columns represent DBD-Del GR expression plasmid. LBD-Del, ligand binding domain-deleted GR; DBD-Del, DNA binding domain-deleted GR. (C) No activation of the ARE-, ERE-, FXRE-, and LXRE-luciferase reporters using different doses of 17-OHP in HepG2 cells. Cells were transfected with various luciferase reporters and the corresponding nuclear receptor expression plasmids. (D) Activation of the PEPCK promoter luciferase reporter in HepG2 cells treated with different doses of 17-OHP for 24 hours. Cells were cotransfected with PEPCK promoter reporters and GR expression plasmids. (E) Relative mRNA levels of PEPCK and TAT in MPHs treated with 17-OHP or vehicle control for 16 hours. (F) Relative mRNA levels of PEPCK and TAT in MPHs treated with 17-OHP (1 $\mu \mathrm{M})$ or 17-OHP plus RU486 (10 $\mu \mathrm{M})$. Cells were pretreated with RU486 for 2 hours and then incubated with 17-OHP for another 16 hours. (C) Subcellular distribution of endogenous GR in MPHs treated with 17-0HP (0.1 $\mu \mathrm{M}$ ) or vehicle control for 1 hour. (H) ChIP assays showing the recruitment of the GR onto the promoter region of the PEPCK and TAT genes. MPHs were treated with 17-OHP $(0.1 \mu \mathrm{M})$ or vehicle control for 1 hour and then subjected to ChIP assays. (I) Coactivator recruitment dose-response curves for $17-0 \mathrm{HP}$ and Dex using a LanthaScreen GR coactivator assay kit. Data are represented as mean $\pm \mathrm{SD}$. ${ }^{* *} P<0.01$, and ${ }^{* * *} P<0.001,1$-way ANOVA followed by the Student-Newman-Keuls test (A, C, D, E, F, and $\mathbf{H}$ ) or 2-tailed unpaired Student's $t$ test (B). 
A

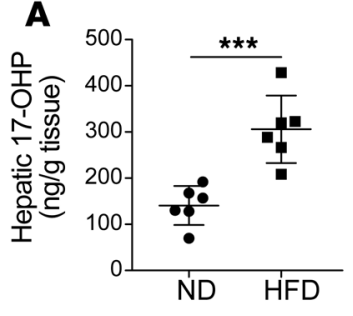

D
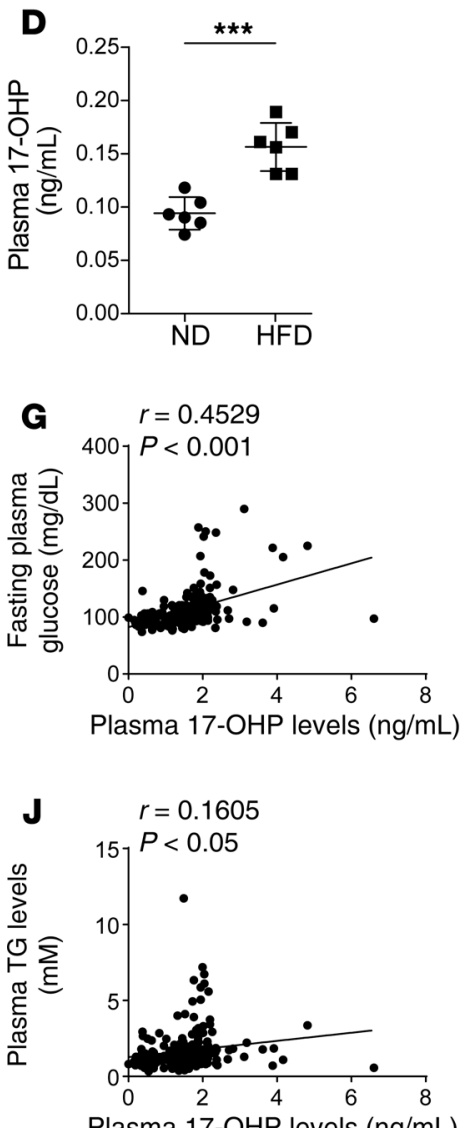

Plasma 17-OHP levels $(\mathrm{ng} / \mathrm{mL})$
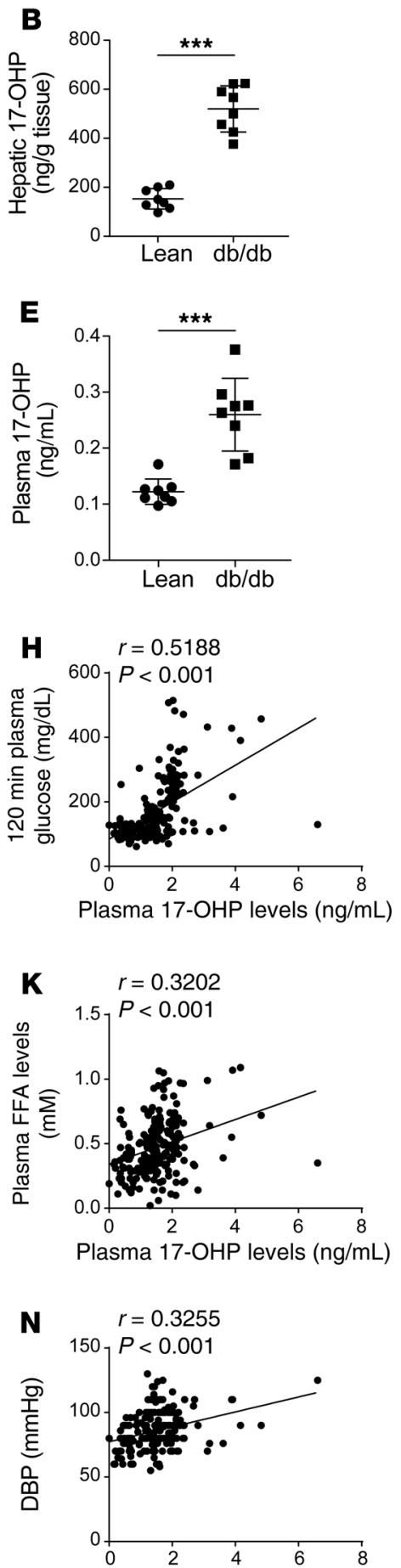

Plasma 17-OHP levels (ng/mL)
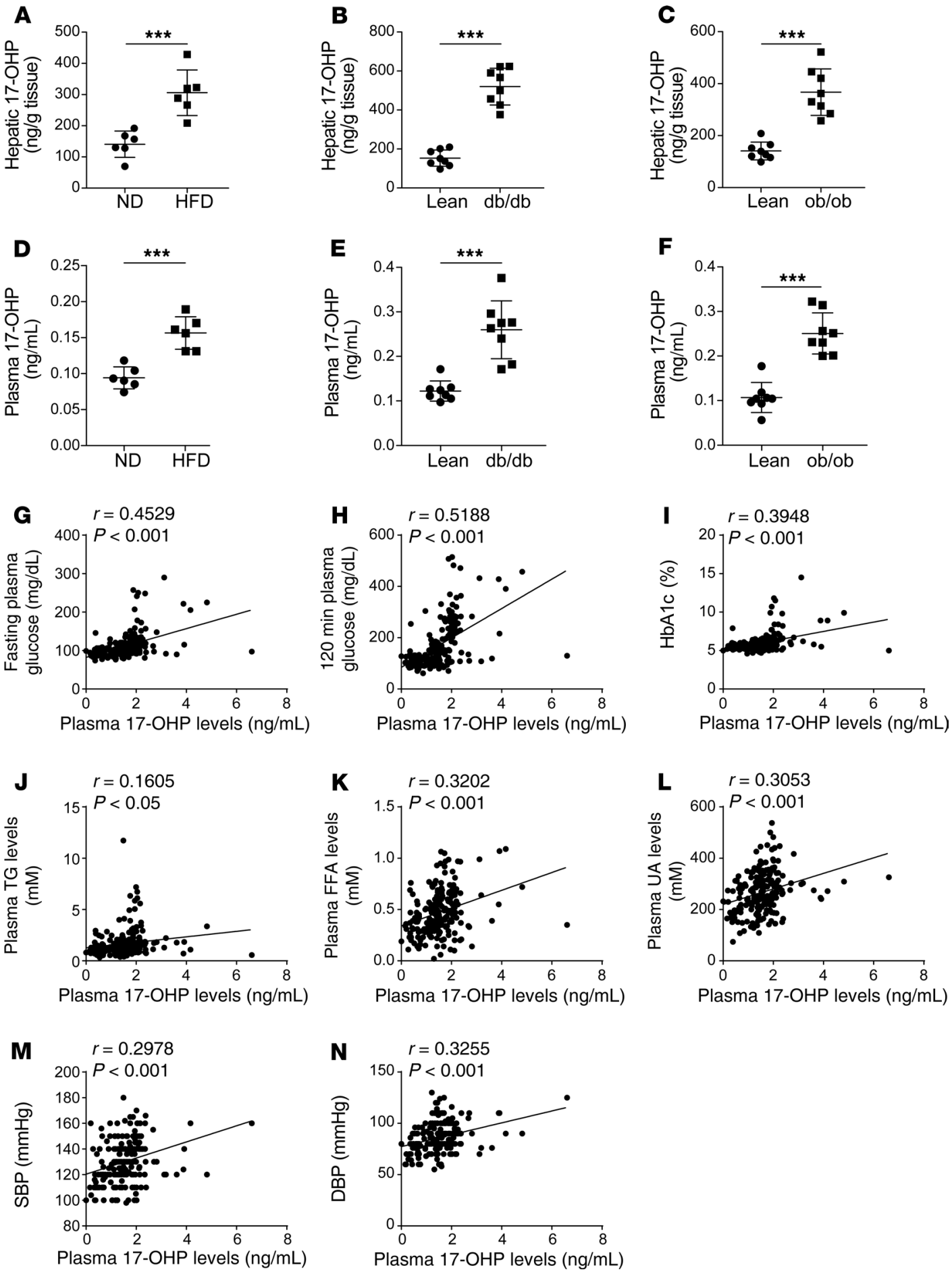

Figure 2. Elevated 17-OHP concentrations in obesity. (A-C) 17-OHP concentrations in the livers of C57BL/ 6 mice fed an HFD or an NCD for 12 weeks (A, $n=6$ per group), $d b / d b$ and lean mice (B, $n=8$ per group), and $o b / o b$ and lean mice (C, $n=8$ per group). (D-F) Plasma 17-OHP levels in 3 types of obese mice. (G-N) Individual correlations between circulating 17-OHP concentrations and fasting plasma glucose levels (C), along with 2-hour postload plasma glucose levels $(\mathbf{H})$, HbA1c (I), plasma TGs (J), FFAs (K), and uric acid (UA) (L), systolic blood pressure (SBP) (M), and diastolic blood pressure (DBP) (N) in humans ( $n=203)$. ${ }^{* * *} P<0.001,2$-tailed unpaired Student's $t$ test $(\mathbf{A}-\mathbf{F})$; Pearson's correlation analysis $(\mathbf{G}-\mathbf{I})$. Data are represented as mean \pm SD.

plasma high-density lipoprotein, low-density lipoprotein, total cholesterol (TC), or urea levels (Supplemental Figure 2, D-G).

We also previously evaluated the clinical characteristics and metabolic profiles in 30 untreated Chinese female subjects with simple virilizing congenital adrenal hyperplasia (SV-CAH) due to 21-hydroxylase deficiency (43). Compared with the normal controls, these patients had significantly higher plasma 17-OHP and lower cortisol levels (43). Further univariate regression analysis showed that plasma 17-OHP levels were positively correlated with fasting plasma insulin levels, homeostatic model assessment for 
Table 1. Clinical characteristics of study participants by diabetic status

\begin{tabular}{|c|c|c|c|c|}
\hline & $\begin{array}{l}\text { Nondiabetes } \\
(\mathrm{n}=72)\end{array}$ & $\begin{array}{l}\text { Prediabetes } \\
(\mathrm{n}=62)\end{array}$ & $\begin{array}{l}\text { Diabetes } \\
(n=69)\end{array}$ & $\begin{array}{l}P \text { value } \\
\text { for trend }\end{array}$ \\
\hline Age, yr & $48.2 \pm 5.1$ & $48.3 \pm 5.3$ & $49.1 \pm 6.0$ & 0.52 \\
\hline Sex (male/female) & $45 / 27$ & $36 / 26$ & $40 / 29$ & 0.61 \\
\hline $\mathrm{BMl}, \mathrm{kg} / \mathrm{m}^{2} 24.4 \pm 3.2$ & $24.4 \pm 3.2$ & $25.1 \pm 3.2$ & $25.7 \pm 2.9$ & 0.058 \\
\hline $\mathrm{WC}, \mathrm{cm}$ & $80.8 \pm 9.2$ & $83.8 \pm 9.1$ & $87.4 \pm 8.8$ & $<0.001$ \\
\hline SBP, $\mathrm{mmHg}$ & $123.4 \pm 15.6$ & $129.3 \pm 14.1$ & $132.8 \pm 17.9$ & 0.0049 \\
\hline $\mathrm{DBP}, \mathrm{mmHg}$ & $80.1 \pm 9.1$ & $84.7 \pm 10.1$ & $84.6 \pm 10.5$ & 0.010 \\
\hline \multicolumn{5}{|l|}{ Lipids } \\
\hline $\mathrm{TC}, \mathrm{mmol} / \mathrm{L}$ & $5.30 \pm 1.17$ & $5.36 \pm 0.98$ & $5.39 \pm 1.06$ & 0.87 \\
\hline $\mathrm{TCs}, \mathrm{mmol} / \mathrm{L}$ & $1.17(0.80-1.57)$ & $1.41(0.90-1.80)$ & $1.73(1.14-2.93)$ & $<0.001$ \\
\hline LDL-C, mmol/L & $3.19 \pm 0.71$ & $3.17 \pm 0.63$ & $3.11 \pm 0.78$ & 0.80 \\
\hline $\mathrm{HDL}-\mathrm{C}, \mathrm{mmol} / \mathrm{L}$ & $1.32 \pm 0.25$ & $1.32 \pm 0.20$ & $1.31 \pm 0.23$ & 0.96 \\
\hline Fasting plasma glucose, $\mathrm{mmol} / \mathrm{L}$ & $5.09 \pm 0.47$ & $5.83 \pm 0.53$ & $7.96 \pm 2.53$ & $<0.001$ \\
\hline Two-hour load plasma glucose, $\mathrm{mmol} / \mathrm{L}$ & $5.83 \pm 0.92$ & $8.40 \pm 1.15$ & $15.94 \pm 4.59$ & $<0.001$ \\
\hline HbA1c, \% & $5.43 \pm 0.32$ & $5.72 \pm 0.42$ & $7.03 \pm 1.86$ & $<0.001$ \\
\hline Uric acid, $\mu \mathrm{mol} / \mathrm{L}$ & $240.4 \pm 73.2$ & $269.0 \pm 78.9$ & $299.2 \pm 80.1$ & $<0.001$ \\
\hline CRP, mg/L & $1.05(0.70-1.90)$ & $1.70(1.00-4.50)$ & $1.70(0.60-4.80)$ & 0.038 \\
\hline $\mathrm{FFA}, \mathrm{mmol} / \mathrm{L}$ & $0.38 \pm 0.14$ & $0.46 \pm 0.20$ & $0.59 \pm 0.24$ & $<0.001$ \\
\hline UACR, mg/g & $1.81(1.25-2.54)$ & $1.37(0.97-1.81)$ & $1.60(1.14-3.08)$ & 0.025 \\
\hline 17- $\mathrm{OHP}, \mathrm{ng} / \mathrm{mL}$ & $1.13 \pm 0.90$ & $1.47 \pm 0.31$ & $2.13 \pm 0.68$ & $<0.001$ \\
\hline
\end{tabular}

Data are presented as the mean $\pm \mathrm{SD}$ or median (interquartile range). Analysis of covariance was performed using general linear models to test the differences among groups, and the $\chi^{2}$ analysis was performed to compare categorical variables. WC, waist circumference; CRP, C-reactive protein. hypothesis that upregulation of Cyp17A1 in the liver is a conserved feature present in both obese mice and humans.

To better determine the pathophysiologic regulation of Cyp17A1 in hepatocytes, we investigated the effects of nutrients on Cyp17A1 expression in Hep1-6 and HepG2 cells. As a result, incubation with palmitic acid (PA) or high concentrations of glucose significantly increased Cyp17A1 expression in both types of cells (Supplemental Figure 4, A-D), suggesting that upregulation of Cyp17A1 in obesity is, at least in part, due to overnutrition.

17-OHP promotes hyperglycemia and insulin resistance in lean mice. To explore the pathophysiological role of 17-OHP in glucose metabolism, we employed different experimental approaches. First, C57BL/6 male mice were given daily i.p. injections of $50 \mathrm{mg} / \mathrm{kg}$ 17-OHP or corn oil (vehicle control) for 14 days. 17-OHP treatment at this dose markedly increased the plasma and hepatic 17-OHP concentrations (Supplemental Figure 5, A and B), and the extent was similar to that observed in obese mice (Figure 2, A-F). However, 17-OHP treatment did not cause severe liver injury, as demonstrated by unaffected plasma alanine aminotransferease (ALT), aspartate insulin resistance (HOMA-IR), plasma TG levels, and systolic blood pressure levels (Supplemental Table 2). Taken together, multiple lines of evidence suggest elevated 17-OHP is associated with the development of insulin resistance and T2DM.

Cyp17A1 expression is increased in the liver of obese mice and humans. 17-OHP is an enzymatic product of the cytochrome P450 family 17 subfamily A member 1 (Cyp17A1) $(44,45)$. Western blot analysis revealed that Cyp17A1 is enriched in testis and also present at lower levels in the liver and inguinal white adipose tissues (iWAT) (Supplemental Figure 3A). Single-cell RNA sequencing further showed that Cyp17A1 is abundantly expressed in hepatocytes compared with other nonparenchymal liver cells (Supplemental Figure 3B).

Quantitative real-time PCR (Real-time qPCR) analysis showed that Cyp17A1 mRNA levels were markedly elevated in the livers of HFD mice (Figure 3A), but not in the testis, iWAT, adrenal gland, brain, or pituitary gland (Figure $3 \mathrm{~A}$ ), compared with mice fed an NCD. The increase in Cyp17A1 expression in the livers of HFD mice was confirmed by Western blotting (Figure 3B). Aberrant upregulation of Cyp17A1 was also observed in the livers of $d b / d b$ and $o b / o b$ mice (Figure 3, C-F). Correlation analysis revealed that changes in the 17-OHP content in the liver closely mirrored the mRNA abundance of Cyp17A1 (Supplemental Figure 3, C-E), suggesting that overproduction of 17-OHP in obese livers is induced by an increased expression of Cyp17A1. Notably, Cyp17A1 mRNA expression was much higher in the livers of obese patients (Figure 3G) and was positively correlated with fasting glucose levels and $\mathrm{HbA1c}$, as determined by Pearson's correlation analysis (Figure 3, H and I). Therefore, our results support the aminotransferase (AST), and bilirubin levels (Supplemental Figure 5, C-E). Body weight and food intake were not affected by 17-OHP treatment (Supplemental Figure 5, F and G), while fed, fasting, and postprandial blood glucose levels were significantly increased (Supplemental Figure 5H). Impaired glucose tolerance and reduced insulin tolerance were observed in 17-OHP-treated mice, which was evidenced by the glucose tolerance test (GTT) and the insulin tolerance test (ITT) (Supplemental Figure 5, I and J). Plasma insulin concentrations were elevated in response to 17-OHP-induced hyperglycemia and insulin resistance (Supplemental Figure 5K). 17-OHP treatment also enhanced hepatic gluconeogenesis and glucose output in the pyruvate tolerance test (PTT) (Supplemental Figure 5L), which was accompanied by increased expression of gluconeogenic enzymes (Supplemental Figure 5M). In addition, 17-OHP-treated mice accumulated a significantly elevated amount of TGs in the liver (Supplemental Figure 5N), while hepatic and plasma TC levels remained unchanged (Supplemental Figure 5, O and P). The deleterious effects of 17-OHP on glucose homeostasis, which included hyperglycemia, hyperinsulinemia, impaired glucose tolerance, reduced insulin tolerance, and enhanced hepatic gluconeogenesis, were also observed in female mice (Supplemental Figure 6, A-F). Therefore, Cyp17A1 may play a similar role in the pathogenesis of metabolic disorders in both male and female sexes. Taken together, our results suggest that long-term administration of 17-OHP could induce insulin resistance and hyperglycemia in lean mice. trol was administered into C57BL/6 mice through tail vein injection. Ad-Cyp17A1 injection resulted in increased protein levels of Cyp17A1
Second, an adenovirus containing the Cyp17A1 gene or GFP con- 
Table 2. Clinical characteristics of participants by quartiles of 17-0HP levels

Variables

Quartiles of 17-0HP

\begin{tabular}{|c|c|c|c|c|c|}
\hline & $\begin{array}{l}\text { Quartile } 1 \\
(n=51)\end{array}$ & $\begin{array}{c}\text { Quartile } 2 \\
(n=51)\end{array}$ & $\begin{array}{c}\text { Quartile } 3 \\
(n=51)\end{array}$ & $\begin{array}{c}\text { Quartile } 4 \\
(n=50)\end{array}$ & \\
\hline 17-OHP, ng/mL & $\leq 1.113$ & $1.113-1.491$ & $1.491-1.923$ & $>1.923$ & $<0.001$ \\
\hline Age, yr & $49.7 \pm 5.7$ & $49.4 \pm 5.7$ & $50.3 \pm 6.3$ & $50.7 \pm 6.0$ & 0.65 \\
\hline Sex (male/female) & $19 / 32$ & $16 / 35$ & $21 / 30$ & $21 / 29$ & 0.43 \\
\hline $\mathrm{BMI}, \mathrm{kg} / \mathrm{m}^{2}$ & $24.5 \pm 3.4$ & $24.5 \pm 2.9$ & $25.2 \pm 3.4$ & $25.7 \pm 2.9$ & 0.18 \\
\hline $\mathrm{WC}, \mathrm{cm}$ & $80.7 \pm 9.9$ & $81.6 \pm 7.9$ & $84.7 \pm 9.6$ & $86.7 \pm 9.0$ & 0.009 \\
\hline $\mathrm{SBP}, \mathrm{mmHg}$ & $124.6 \pm 15.2$ & $126.0 \pm 17.4$ & $128.5 \pm 14.4$ & $131.1 \pm 18.1$ & 0.26 \\
\hline $\mathrm{DBP}, \mathrm{mmHg}$ & $80.6 \pm 9.3$ & $82.2 \pm 10.8$ & $83.7 \pm 10.5$ & $83.7 \pm 9.4$ & 0.40 \\
\hline \multicolumn{6}{|l|}{ Lipids } \\
\hline $\mathrm{TC}, \mathrm{mmol} / \mathrm{L}$ & $5.30 \pm 1.11$ & $5.26 \pm 1.18$ & $5.37 \pm 1.03$ & $5.45 \pm 1.02$ & 0.81 \\
\hline $\mathrm{TGs}, \mathrm{mmol} / \mathrm{L}$ & $\begin{array}{c}1.22 \\
(0.87-1.56)\end{array}$ & $\begin{array}{c}1.33 \\
(0.90-1.78)\end{array}$ & $\begin{array}{c}1.56 \\
(1.00-1.97)\end{array}$ & $\begin{array}{c}1.63 \\
(1.09-2.29)\end{array}$ & 0.02 \\
\hline LDL-C, mmol/L & $3.19 \pm 0.65$ & $3.13 \pm 0.77$ & $3.14 \pm 0.63$ & $3.19 \pm 0.79$ & 0.95 \\
\hline $\mathrm{HDL}-\mathrm{C}, \mathrm{mmol} / \mathrm{L}$ & $1.32 \pm 0.25$ & $1.30 \pm 0.24$ & $1.32 \pm 0.20$ & $1.32 \pm 0.24$ & 0.99 \\
\hline Fasting plasma glucose, $\mathrm{mmol} / \mathrm{L}$ & $5.26 \pm 0.73$ & $5.66 \pm 0.62$ & $6.13 \pm 1.55$ & $7.59 \pm 2.76$ & $<0.001$ \\
\hline Two-hour load plasma glucose, $\mathrm{mmol} / \mathrm{L}$ & $6.37 \pm 2.17$ & $7.51 \pm 1.72$ & $9.93 \pm 4.52$ & $14.49 \pm 5.79$ & $<0.001$ \\
\hline HbA1c, \% & $5.50 \pm 0.45$ & $5.59 \pm 0.42$ & $5.91 \pm 0.83$ & $6.94 \pm 1.99$ & $<0.001$ \\
\hline Uric acid, $\mu \mathrm{mol} / \mathrm{L}$ & $247.8 \pm 66.1$ & $255.0 \pm 84.6$ & $272.0 \pm 82.3$ & $290.6 \pm 82.7$ & 0.035 \\
\hline CRP, mg/L & $\begin{array}{c}1.35 \\
(0.90-2.40)\end{array}$ & $\begin{array}{c}1.65 \\
(0.90-4.50)\end{array}$ & $\begin{array}{c}1.20 \\
(0.60-3.80)\end{array}$ & $\begin{array}{c}1.70 \\
(0.60-4.90)\end{array}$ & 0.081 \\
\hline $\mathrm{FFA}, \mathrm{mmol} / \mathrm{L}$ & $0.39 \pm 0.16$ & $0.44 \pm 0.19$ & $0.49 \pm 0.21$ & $0.54 \pm 0.24$ & 0.002 \\
\hline UACR, $\mathrm{mg} / \mathrm{g}$ & $\begin{array}{c}1.64 \\
(1.23-2.71)\end{array}$ & $\begin{array}{c}1.64 \\
(1.12-2.29)\end{array}$ & $\begin{array}{c}1.31 \\
(1.05-2.26)\end{array}$ & $\begin{array}{c}1.58 \\
(1.14-2.83)\end{array}$ & 0.028 \\
\hline
\end{tabular}

Data are presented as the mean \pm SD or median (interquartile range). ANOVA was performed using general linear models to test the differences among groups, and the $\chi^{2}$ analysis was performed to compare categorical variables.

Cyp17A1 or treated with 17-OHP (Supplemental Figure 7, F-I). This result is consistent with a previous study showing that treatment of mice with 17-OHP at a dose of $125 \mathrm{mg} / \mathrm{kg}$ did not affect circulating and hepatic bile acid levels (46). Therefore, the metabolic effects of 17-OHP are likely independent of changes in bile acid levels.

We also evaluated the potential physiological role of Cyp17A1 in the liver. We measured the expression of Cyp17A1 in normal C57BL/6 mice during fed, fasting, and refed conditions. As a result, Cyp17A1 expression was activated after a period of fasting and suppressed upon refeeding (Supplemental Figure 8, A and B), which correlates well with the characteristic regulatory pattern of gluconeogenic genes (Supplemental Figure 8, C and D). Cyp17A1 levels in a fasted state are less than those detected in obese mice (Figure 3, A-D). These results suggest that Cyp17A1 might be involved in the regulation of hepatic gluconeogenesis in normal mice. C57BL/6 mice were then transfected with the Cyp17A1 adenovirus at a low dose $\left(5 \times 10^{8}\right.$ PFUs per mouse). This low dose of Ad-Cyp17A1 moderately increased Cyp17A1 protein

in the livers of C57BL/6 mice relative to the Ad-GFP control group (Figure 4A). In contrast, protein levels of Cyp17A1 in the WAT, brown adipose tissue, and skeletal muscle were not affected by the Cyp17A1 adenovirus (Supplemental Figure 7A). The 17-OHP concentrations in the liver and plasma were also increased as expected (Figure 4, B and C). Analysis of the blood glucose levels of mice showed Ad-Cyp17A1 significantly increased blood glucose levels in both fed and fasting states (Figure 4D). GTTs and ITTs revealed an impaired glucose disposal rate and reduced insulin sensitivity in Cyp17A1-expressing mice (Figure 4, E and F). Consistently, circulating insulin levels were higher in Cyp17A1-expressing mice (Figure 4G). Phosphorylated AKT, which plays a central role in insulin signaling, was reduced in Cyp17A1-injected mice versus Ad-GFP controls (Figure 4H). PTTs also confirmed Ad-Cyp17A1 enhanced gluconeogenesis and hepatic glucose output (Figure 4I). In agreement, gluconeogenic enzymes were also increased (Figure 4J). Analysis of lipid metabolism showed that mice expressing Ad-Cyp17A1 had a significantly higher TG content in the liver (Figure 4, K and L). Food intake and body weight were not altered (data not shown). In addition, plasma adrenocorticotropic hormone (ACTH) and corticosterone levels were reduced in mice treated with 17-OHP or mice overexpressing Cyp17A1 (Supplemental Figure 7, B-E). The reduction of ACTH and corticosterone levels could be attributed to a feedback suppression, which further supports the notion that 17-OHP is an activator of GR. Of note, plasma and hepatic bile acid levels were unaltered in mice overexpressing levels in the liver (Supplemental Figure 8E), which was similar to the extent of the expression changes observed in the fasted state. Blood glucose levels and expression of gluconeogenic enzymes were moderately increased in Cyp17A1-overexpressing mice (Supplemental Figure 8, F and G). In contrast, insulin sensitivity was not significantly altered, as determined by ITT (Supplemental Figure $8 \mathrm{H})$. Therefore, our results indicate that hepatic Cyp17A1 in healthy mice plays a physiological role in maintaining glucose homeostasis under fasting conditions.

Third, to avoid immune responses triggered by the adenovirus, an adeno-associated virus (AAV) using a liverspecific thyroxine-binding globulin promoter was used. AAV-Cyp17A1 injection increased protein levels of Cyp17A1 in the liver of C57BL/6 mice (Supplemental Figure 9A), but not in other metabolic tissues examined (Supplemental Figure 9B). Hepatic and plasma 17-OHP concentrations were increased in the AAV-Cyp17A1 group compared with the AAV-GFP group (Supplemental Figure 9, C and D). Measurement of blood glucose levels at fed and fasting states showed that Cyp17A1-expressing mice had higher blood glucose levels (Supplemental Figure 9E). Furthermore, plasma insulin levels were also markedly increased (Supplemental Figure 9F). GTT and ITT assays revealed a significantly reduced glucose and insulin tolerance in Cyp17A1-expressing mice (Supplemental Figure 9, G and $\mathrm{H}$ ). Hepatic glucose production, 

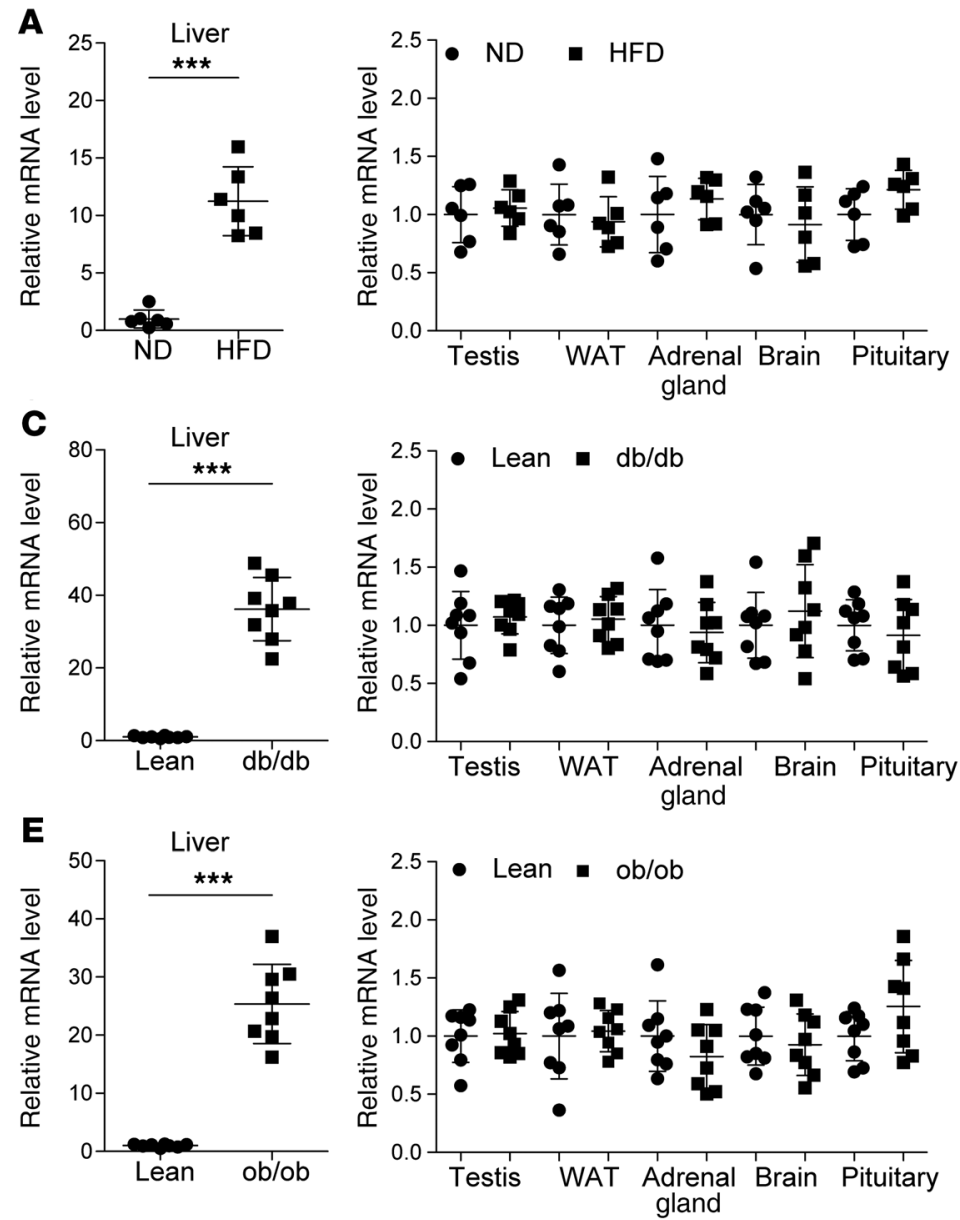

$\mathbf{F}$
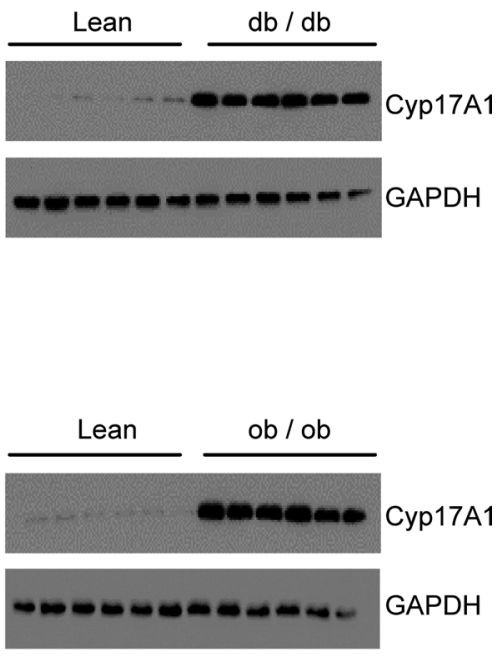
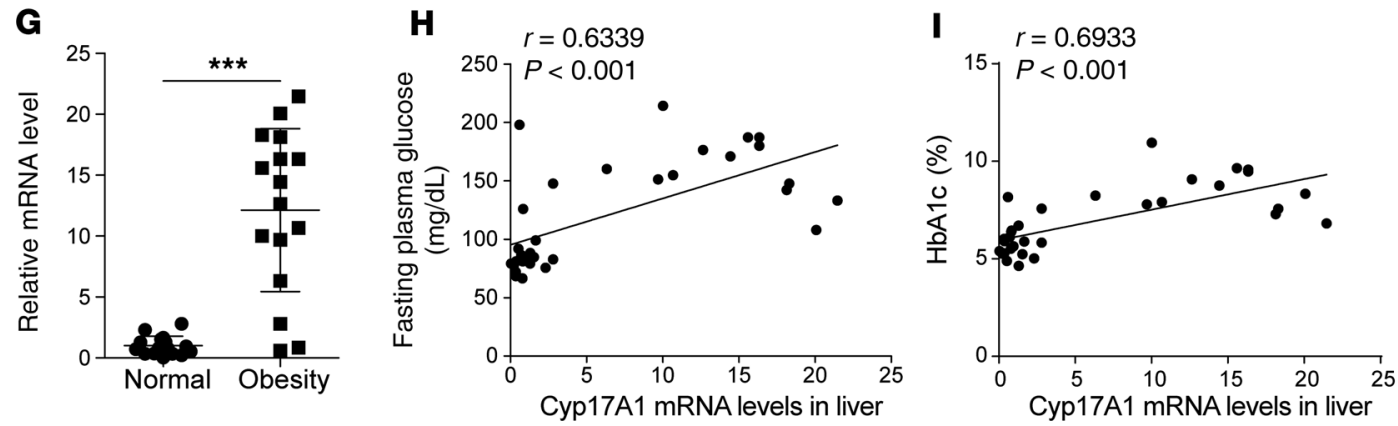

Figure 3. Increased Cyp17A1 expression in obese livers. (A) Relative mRNA levels of Cyp17A1 in the liver, testis, iWATs, adrenal gland, brain, and pituitary gland of mice fed an HFD or an NCD for 12 weeks. $n=6$ per group. (B) Western blots of total liver lysates from mice fed NCD or HFD diet. (C) Relative mRNA levels of Cyp17A1 in the liver, testis, iWATs, adrenal gland, brain, and pituitary gland of lean and $d b / d b$ mice. $n=8$ per group. (D) Western blots of total liver lysates from lean and $d b / d b$ mice. (E) Relative mRNA levels of Cyp17A1 in the liver, testis, iWATs, adrenal gland, brain, and pituitary gland of lean and $o b / o b$ mice. $n=8$ per group. (F) Western blots of total liver lysates from lean and ob/ob mice. (C) Relative mRNA levels of Cyp17A1 in the livers of obese and normal weight humans. $n=16$ per group. ( $\mathbf{H}$ and $\mathbf{I})$ Association between relative Cyp17A1 mRNA levels and fasting plasma glucose levels $(\mathbf{H})$ and $\mathrm{HbA1c}(\mathbf{I})$ in humans $(n=32) .{ }^{* *} P<0.001$, 2-tailed unpaired Student's $t$ test $(\mathbf{A}, \mathbf{C}, \mathbf{E}$, and $\mathbf{G})$ or Pearson's correlation analysis $(\mathbf{H}$ and $\mathbf{I})$. Data are represented as mean \pm SD.

estimated by PTT, was also higher in mice injected with AAV-Cyp17A1thaninmiceinjected withAAV-GFP(Supplemental Figure 9I). Increased hepatic TG contents were also observed in AAV-Cyp17A1-injected mice (Supplemental Figure 9J).

To examine the functions of the hepatic GR in mediating the effects of 17-OHP in vivo, C57BL/6 mice were depleted of endogenous GR expression using adenoviral shRNA (sh-GR) and then treated with 17-OHP or vehicle control (Figure 5A). As a result, suppression of hepatic GR expression substantially blocked the metabolic side effects caused by 17-OHP, including hyperglycemia, hyperinsulinemia, hepatosteatosis, insulin resistance, glucose intolerance, and enhanced gluconeogenesis (Figure 5, $\mathrm{B}-\mathrm{H})$. Therefore, these results support the idea that hepatic GR mediates the effects of 17-OHP on glucose metabolism in mice.

Knockdown of hepatic Cyp17A1 improves obesity-associated metabolic disorders. Finally, we performed RNAi knockdown in the liver of $d b / d b$ mice using 2 independent recombinant adenovirus-expressing shRNAs directed toward Cyp17A1 
A
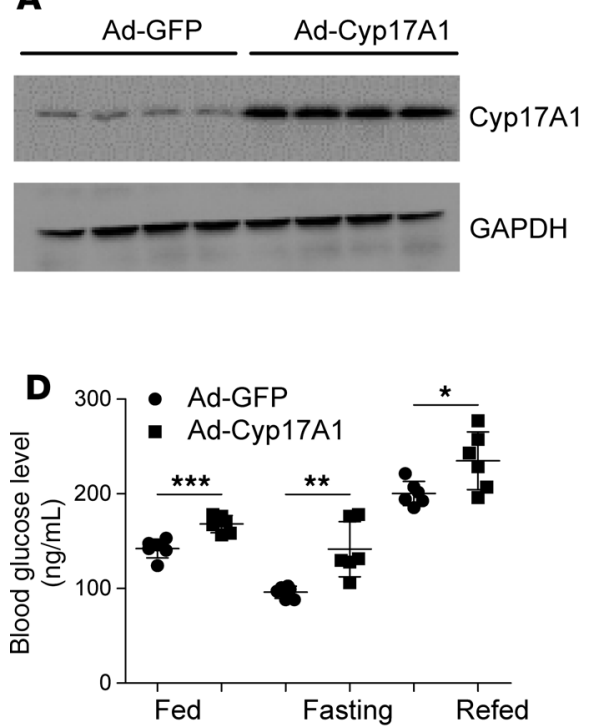
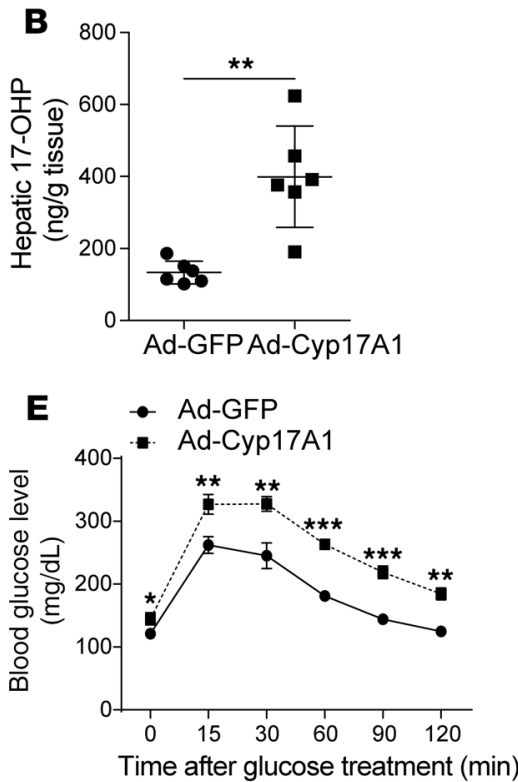
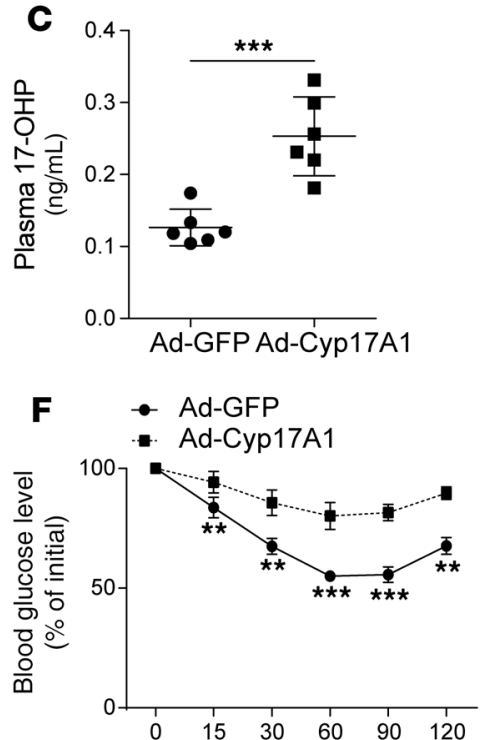

Time after insulin treatment (min)

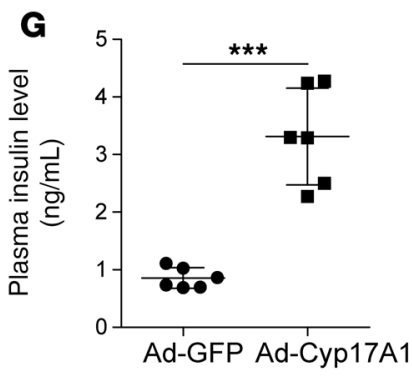

H

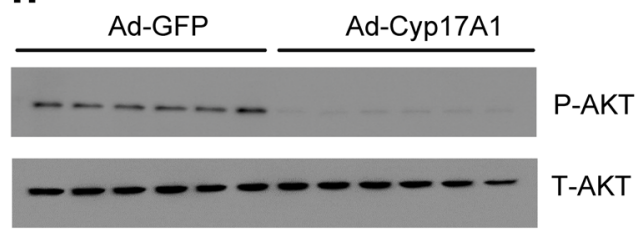

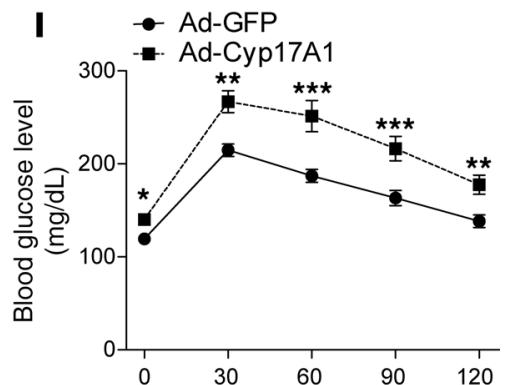

Time after pyruvate treatment (min)
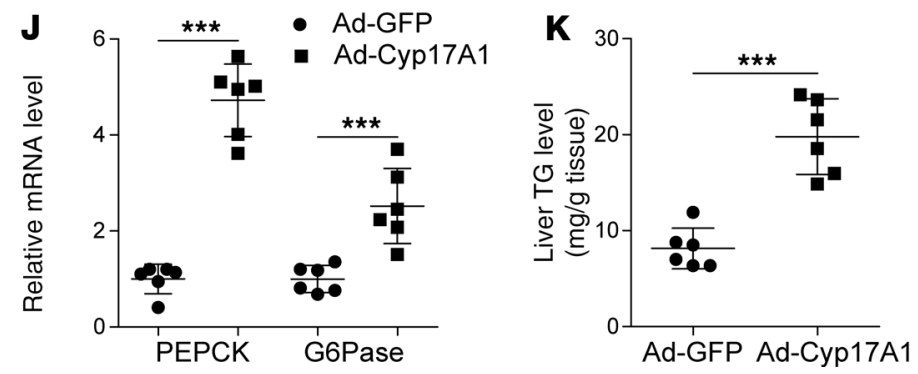

L Ad-GFP
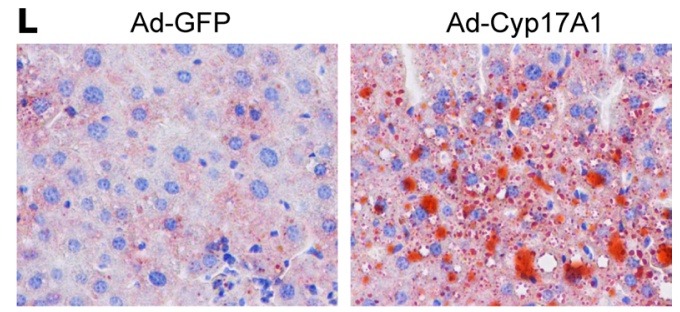

Figure 4. 17-0HP promotes hyperglycemia and insulin resistance in vivo. (A) Western blots showing the protein levels of Cyp17A1 in the livers of C57BL/6 mice transfected with adenovirus containing Cyp17A1 or GFP. (B and C) 17-OHP concentrations in the livers and plasma from mice overexpressing Cyp17A1 or GFP. (D-C) Blood glucose levels on postinjection day 5 (D), GTTs and ITTs on postinjection days 7 and 10, respectively (E and F), and plasma insulin levels (C) in 2 groups of mice. (H) Western blots showing the protein levels of phosphorylated AKT (serine 473) and total AKT in the livers of mice overexpressing Cyp17A1 or GFP. (I) PTTs in the 2 groups of mice on postinjection day 13. (J) Relative mRNA levels of PEPCK and G6Pase in the liver. (K) Liver TG contents. (L) Oil red 0 -stained liver sections. Original magnification, $\times 200.2 \times 10^{9}$ PFUs per mouse were delivered into C57BL/ 6 mice through the tail vein. Sixteen days later, mice were sacrificed for analysis $(\mathbf{A}-\mathbf{C}, \mathbf{G}, \mathbf{H}$, and $\mathbf{J}-\mathbf{L}) . n=6$ per group. Data are represented as mean \pm SD. ${ }^{*} P<0.05 ;{ }^{*} P<0.01 ;$ and ${ }^{* * *} P<0.001$, 2-tailed unpaired Student's $t$ test (B-D, G, J, and K); 1-way ANOVA followed by Student-Newman-Keuls test (E, F, and I).

(C1 and C2). As a result, Cyp17A1 mRNA and protein levels were significantly decreased relative to the negative control (NC) following the injection of both shRNA adenoviruses (Figure 6, A and B). Hepatic and circulating 17-OHP levels were also significantly reduced (Figure 6, C and D). Although body weight and food intake remained similar among the 3 groups (data not shown), the blood glucose levels and plasma insulin concentrations were decreased in the knockdown mice (Figure 6, E and F). These observations are consistent with improved glucose tolerance, enhanced insulin tolerance, and decreased hepatic glucose production (HGP), as determined by GTT, ITT, and PTT assays (Figure 6, G-I). Consistently, AKT phosphorylation was significantly increased while expression levels of gluconeogenetic genes were reduced (Figure $6, \mathrm{~J}$ and $\mathrm{K}$ ). In addition, the hepatic TG contents were also reduced in the livers of Cyp17A1-knockdown mice (Figure 6, L and M). Additionally, plasma ALT and AST levels were significantly reduced (Figure 6, $\mathrm{N}$ and $\mathrm{O}$ ), suggesting that suppression of hepatic Cyp17A1 has beneficial effects on whole-body metabolism. 
A

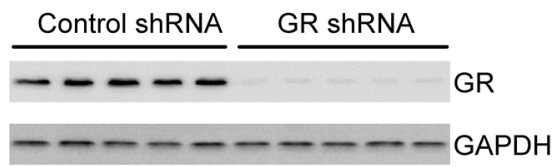

E

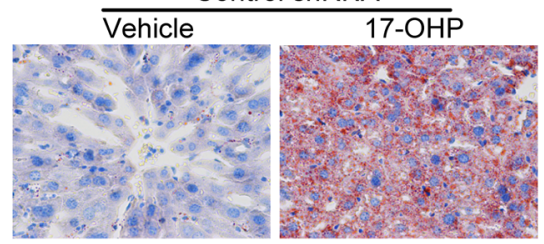

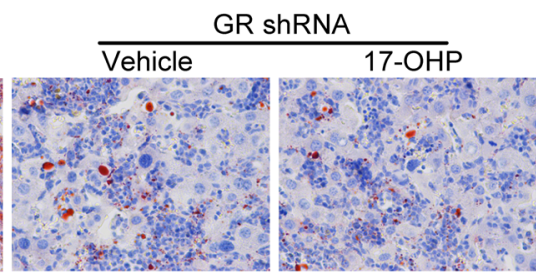

B

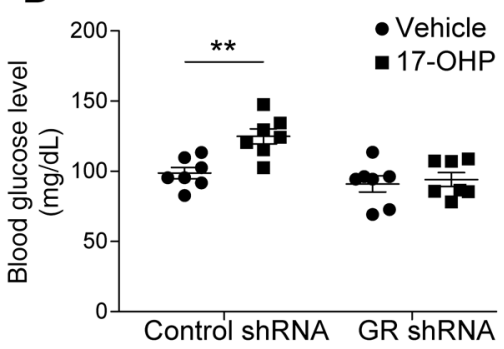

F

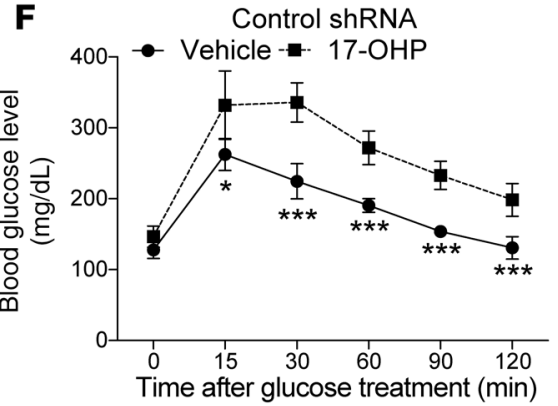

G

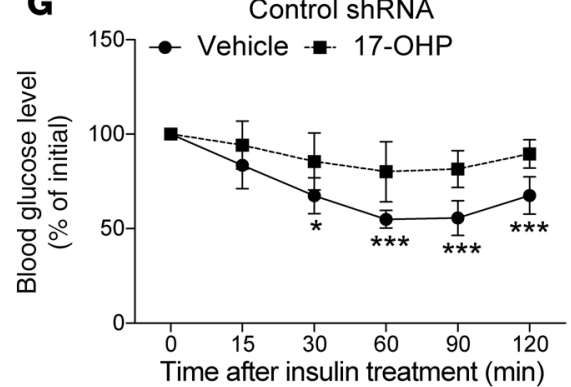

H

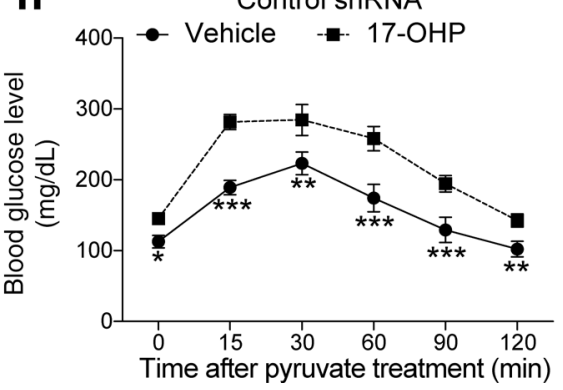

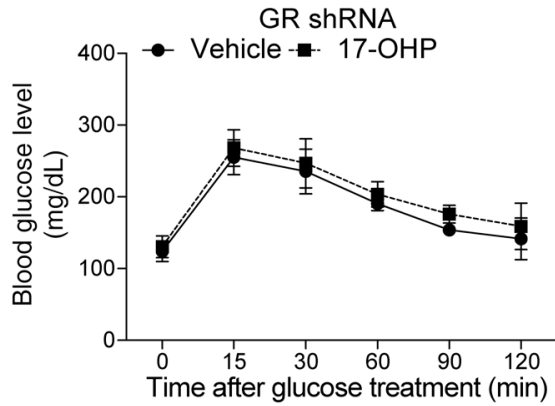

GR shRNA

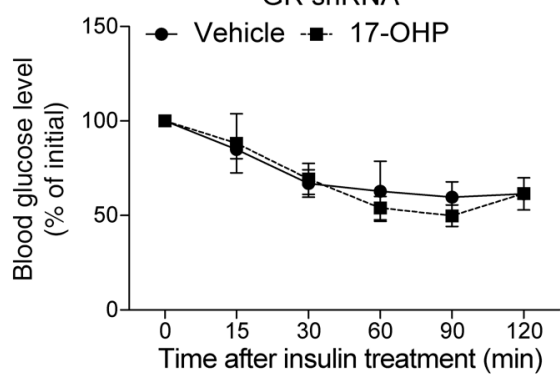

GR shRNA

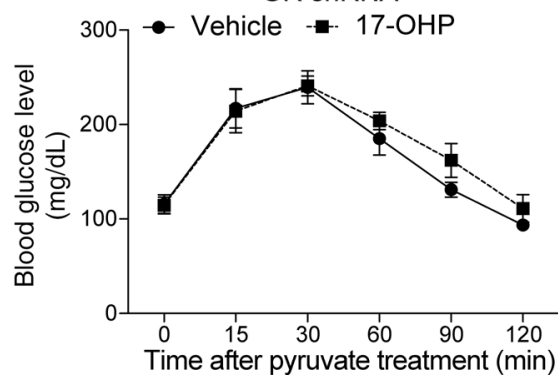

Figure 5. Ablation of hepatic GR attenuates 17-0HP-induced metabolic side effects. (A) Western blot results showing GR protein levels in the livers of mice injected with adenoviral shRNA targeting CR or the NC. (B-D) Blood glucose levels on postinjection day 8 (B), plasma insulin levels (C), and hepatic TC content (D). (E) Oil red 0 staining of liver sections. Original magnification, $\times 200$. (F-H) GTTs (F), ITTs (G), and PTTs (H) on postinjection days 10 , 12, and 14 , respectively. C57BL/6 mice were injected with GR shRNA or control shRNA at a dose of $4 \times 10^{9} \mathrm{PFUs}$ per mouse through the tail vein. On postinjection day 2, mice were given 17-OHP or corn oil (vehicle) daily at doses of $50 \mathrm{mg} / \mathrm{kg}$ body weight by i.p. injection for another 14 days. Then mice were sacrificed for analysis (A, C-E). $n=7$ per each subgroup. Data are represented as mean \pm SD. ${ }^{*} P<0.01$, and ${ }^{* * *} P<0.001,1$-way ANOVA followed by the Student-Newman-Keuls test (B-D and F-H).

\section{Discussion}

Previous studies have shown Cyp17A1 is expressed in the liver and that the extreme induction of Cyp17A1 in hepatocytes can contribute to liver injury in FXR/SHP double-knockout mice (46). However, its pathophysiological functions in glucose metabolism have not been fully explored. In this report, we found that obesity-associated upregulation of Cyp17A1 in the liver led to elevated hepatic and circulating levels of 17-OHP. We used gain- and loss-of-function approaches to explore whether Cyp17A1 and 17-OHP have deleterious effects on glucose homeostasis. To our knowledge, we are the first to show that elevated plasma 17-OHP levels are associated with T2DM in human subjects. Therefore, our present study supports the hypothesis that 17-OHP in the liver is an important causal factor in the development of metabolic disorders. Interestingly, recent studies suggest that local GCs' action due to upregulated activity of 11ß-hydroxysteroid dehydrogenase type $1(11 \beta$-HSD1) in the liver of obese mice contributes to the pathogenesis of metabolic disorders $(47,48)$. In addition, some animal studies have shown the metabolic benefits of $11 \beta$-HSD1 inhibitors, although careful consideration and more clinical data are still required in T2DM patients $(49,50)$. Together with these reports, our findings presented herein arise from a shift in our rethinking about the roles of circulating GCs in the development of metabolic diseases. 

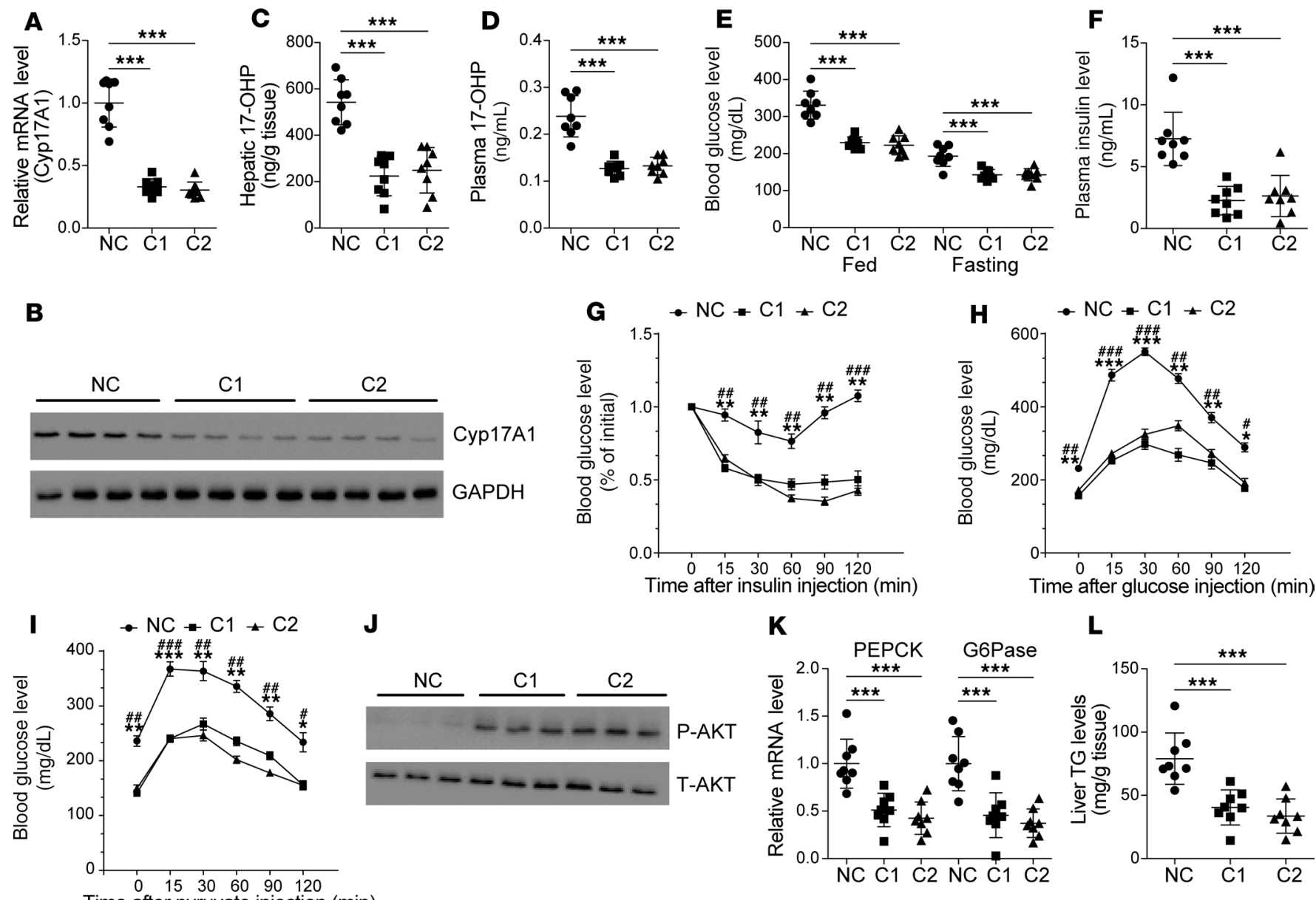
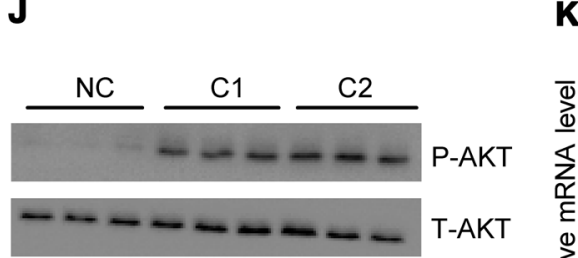

$\mathbf{K}$

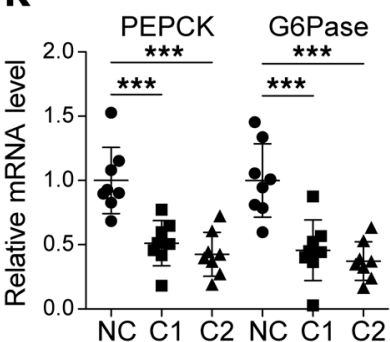

$\mathbf{L}$

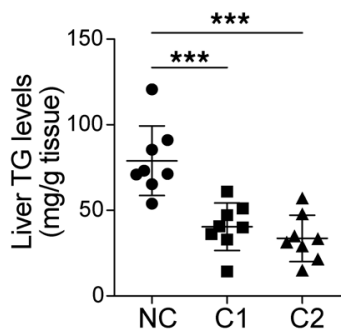

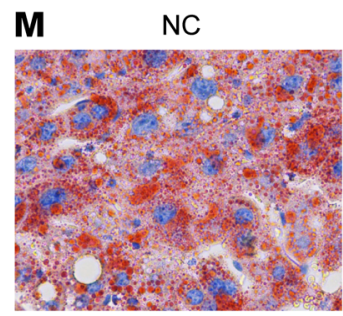
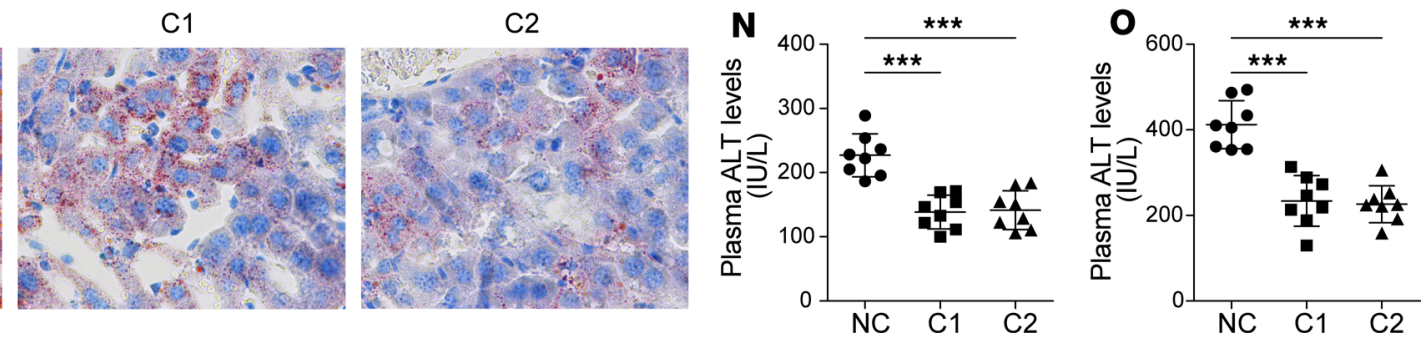

Figure 6. Knockdown of hepatic Cyp17A1 improves hyperglycemia in obese mice. (A and B) Relative mRNA levels (A) and protein levels (B) of Cyp17A1 in the $d b / d b$ mice injected with adenovirus shRNA targeting Cyp17A1 (C1 and C2) or NC. (C and D) Hepatic and circulating 17-OHP concentrations in the 3 groups of $d b / d b$ mice. (E and F) Blood glucose levels on postinjection day 5 and plasma insulin levels in the 3 groups of mice. (G-I) ITTs (G), GTTs (H), and PTTs (I) on postinjection days 7, 10, and 13, respectively. (J) Western blots of protein levels of phosphorylated AKT (Serine 473) and total AKT in livers of mice. (K) Relative mRNA levels of gluconeogenic genes in livers of 3 groups of mice. (L) Hepatic TC content. (M) Representative oil red 0 staining. Original magnification, $\times 200$. $(\mathbf{N}-\mathbf{0})$ Plasma ALT $(\mathbf{N})$ and AST (0) levels in the 3 groups of mice. $n=8$ per group. $4 \times 10^{9}$ PFUs per mouse were delivered into $d b / d b$ mice through the tail vein. Sixteen days later, mice were sacrificed for analysis $(\mathbf{A}-\mathbf{D}, \mathbf{F}$, and J-0). Data are represented as mean $\pm \mathrm{SD}$. ${ }^{* * *} P<0.001,1$-way ANOVA followed by the Student-Newman-Keuls test $(\mathbf{A}, \mathbf{C}-\mathbf{F}, \mathbf{K}, \mathbf{L}, \mathbf{N}$, and $\mathbf{0}) .{ }^{*} P<0.05 ;{ }^{*} P<0.01 ;$ ${ }^{* * *} P<0.001$, NC versus $\mathrm{C} 1(\mathbf{G}-\mathrm{I}) .{ }^{\#} P<0.05 ;{ }^{\# \#} P<0.01 ;{ }^{\# \#} P<0.001$, NC versus $\mathrm{C} 2$ (G-I).

In the present study, our data showed that $17-\mathrm{OHP}$ at a concentration of $0.1 \mu \mathrm{M}$ can enhance the transcriptional activity of GR, promote its nuclear translocation, and upregulate the expression of its target genes (Figure 1, A, B, D, E, G, and $\mathrm{H})$. In addition, we found that the concentration of 17-OHP in mouse livers ranged from 100 to $600 \mathrm{ng} / \mathrm{g}$ tissue, which corresponds to a concentration of 0.3 to $1.8 \mu \mathrm{M}$. Thus, hepatic levels of 17-OHP, which are much higher than the amount necessary to upregulate GR target genes, are sufficient to activate GR and contribute to the pathogenesis of metabolic disorders. In addition, our animal experiments showed that 17-OHP injection at a dose of $50 \mathrm{mg} / \mathrm{kg}$ increased hepatic 17-OHP concentrations by 2.6-fold (Supplemental Figure 5B). The extent of this rise is similar to that observed in 3 different obese mouse models (Figure 2, A-C), which demonstrates that hepatic overproduction of 17-OHP can disrupt glucose homeostasis. 
Our knockdown experiments in $d b / d b$ mice suggest that suppression of Cyp17A1 might be a therapeutic avenue for treating hyperglycemia and diabetes (Figure 6, A-M). To further validate this hypothesis, abiraterone, a selective inhibitor of Cyp17A1 prescribed in patients with metastatic castration-resistant prostate cancer (51-53), was used. Ten-week-old male $d b / d b$ mice were treated daily with abiraterone $(0.5 \mathrm{mmol} / \mathrm{kg} / \mathrm{d})$ or vehicle control by i.p. injection for 30 days. This dose was chosen based on previous studies $(54,55)$. As a result, abiraterone treatment decreased blood glucose levels and plasma insulin levels in $\mathrm{db} / \mathrm{db}$ mice, compared with vehicle control (Supplemental Figure 10, A and B). Furthermore, improved glucose tolerance, enhanced insulin sensitivity, decreased expression levels of gluconeogenetic genes, and reduced hepatic TG accumulation were observed in $d b / d b$ mice treated with abiraterone (Supplemental Figure 10, C-F). Interestingly, a recent case report showed that abiraterone therapy may be associated with hypoglycemia when administered to patients with prostate cancer (56), further emphasizing the potent glucoselowering effects of Cyp17A1 inhibitors. However, due to ubiquitous expression of Cyp17A1 in many tissues with enrichment in the testis shown in this study (Supplemental Figure 3A) and other reports, abiraterone can block androgen biosynthesis $(57,58)$. Therefore, compounds that specifically target hepatic Cyp17A1 would offer a more ideal way for treating hyperglycemia and insulin resistance without changes in other steroid hormones.

17- $\alpha$ Hydroxyprogesterone caproate has been shown to reduce the rate of recurrent preterm delivery in pregnant women and is approved for the prevention of preterm birth by the US Food and Drug Administration (59, 60). However, subsequent studies examining the association between supplemental $17-\alpha$ hydroxyprogesterone caproate and gestational diabetes mellitus (GDM) uncovered a significantly increased rate of GDM in pregnant women treated with 17- $\alpha$ hydroxyprogesterone caproate (61-63). Furthermore, 2 meta-analyses, one including 6 and the other including 11 studies, also clearly demonstrated that women receiving 17- $\alpha$ hydroxyprogesterone caproate had a higher risk of developing GDM, while women receiving progesterone may have had a decreased risk $(64,65)$. Here, we showed that 17-OHP treatment also increased blood glucose levels, reduced insulin tolerance, and enhanced hepatic glucose production in female mice (Supplemental Figure 6, A-F). Therefore, our results may help to explain the increased incidence of GDM in women receiving 17- $\alpha$ hydroxyprogesterone caproate.

There are several limitations of this study that we would like to point out. First, the sample size of the human study was relatively small. Multicenter prospective studies are needed to further establish the association of plasma 17-OHP levels and incidence of T2DM. Second, recent studies have shown the GR in adipocytes also exerts important roles in the development of metabolic disorders $(66,67)$. Understanding whether overproduction of hepatic 17-OHP could activate the adipocyte GR and subsequently affect glucose and lipid metabolism requires further in-depth analysis. Third, the development and studies of hepatocyte-specific Cyp17A1-knockout mice are still needed to confirm the role of Cyp17A1 in systemic glucose metabolism under different energetic states.

In summary, our results have identified a hepatic Cyp17A1/17$\mathrm{OHP} / \mathrm{GR}$-dependent pathway that plays a role in the develop- ment of hyperglycemia and insulin resistance. Strategies that specifically target Cyp17A1 in the liver may therefore have beneficial effects for treating T2DM and related metabolic disorders.

\section{Methods}

Reagents. 17-OHP, DHEAS, deoxycorticosterone, estrone, pregnenolone, and progesterone were purchased from APExBIO Technology LLC. Bovine serum albumin, Dex, estradiol (E2), glucose, GW4064, PA, R1881, RU486, and T0901317 were purchased from MilliporeSigma. Abiraterone was provided by Zhenfei Li (Shanghai Institute of Biochemistry and Cell Biology, Chinese Academy of Sciences, Shanghai, China).

Human studies. A total of 203 subjects of Chinese Han descent were recruited from community-based epidemiological studies of diabetes and related metabolic disorders. Detailed information concerning this study population has been previously described (40-42). Briefly, all the subjects underwent anthropometric measurements and a $75 \mathrm{~g}$ oral glucose tolerance test (OGTT) in the morning. Blood samples were obtained at 0 and 120 minutes of the OGTT. Plasma glucose levels were measured using the glucose oxidase method. HbA1c levels were determined using high-performance liquid chromatography (Bio-Rad). Concentrations of lipid profiles were measured on a Hitachi 7600-020 automated biochemical analyzer; these included TC, TGs, high-density lipoprotein cholesterol (HDL-C), low-density lipoprotein cholesterol (LDL-C), and FFA. High sensitive C-reactive protein (hsCRP) levels were measured by using CardioPhase hsCRP reagent in a particle-enhanced immunoturbidimetric assay (Dade Behring). Urine albumin-to-creatinine ratio (UACR) was calculated as urine albuminuria/creatinine. In addition, a total of 60 subjects with SV-CAH and normal subjects ( $n=30$ per each group) were recruited as previously described (43). For the analysis of hepatic Cyp17A1 mRNA expression, a liver biopsy was performed in subjects who donated their partial livers for liver transplantation, as previously described (68).

Animal studies. Male $d b / d b$ and $o b / o b$ mice 8 weeks of age were purchased from the Nanjing Biomedical Research Institute of Nanjing University (Nanjing, China). Male C57BL/6 mice 8 to 10 weeks of age and female C57BL/6 mice 8 weeks of age were obtained from the Shanghai Laboratory Animal Company (SLAC). To generate HFDinduced obese mice, C57BL/6 mice were fed ad libitum with an NCD or an HFD (D12492; Research Diets) for 12 weeks. All mice were housed at $21^{\circ} \mathrm{C} \pm 1^{\circ} \mathrm{C}$ with $55 \% \pm 10 \%$ humidity and a 12 -hour light/12-hour dark cycle. The metabolic features of HFD-fed, ob/ob, and $d b / d b$ mice were previously described (68). 17-OHP or corn oil (vehicle control) was administered daily at a dose of $50 \mathrm{mg} / \mathrm{kg}$ body weight by i.p. injection for 14 days. Plasma ALT and AST levels were measured using kits from Shanghai KeHua Bio-Engineering Company. Oil red O staining of liver sections was conducted in formalin-fixed and sucrose-protected tissues. For analyzing hepatic TG content, liver tissues ( 100 mg) were homogenized in $1 \mathrm{~mL}$ of $5 \% \mathrm{NP}-40$ solution and heated to $100^{\circ} \mathrm{C}$ and then cooled to room temperature. The tissue homogenates were centrifuged for 2 minutes, and the supernatants were processed to measure TG content using the Triglyceride Quantification Kit (BioVision).

Adenovirus and AAV. Recombinant adenoviruses for the overexpression or knockdown of target genes have been described previously (68). Briefly, full-length mouse Cyp17A1 cDNA or the GFP gene was cloned into GV314 adenoviral vector (CMV-MCS-3FLAG-SV-40EGFP) from GeneChem, and $2 \times 10^{9}$ PFUs per mouse were delivered into C57BL/ 6 mice for 16 days. To produce AAV, the Cyp17A1 or 
GFP sequence was cloned into the GV632 vector (TBGp-MCS-SV-40 PolyA) from GeneChem, in which the thyroid hormone-binding globulin promoter controls hepatocyte-specific expression of Cyp17A1, and $5 \times 10^{10}$ PFUs per mouse were delivered into C57BL/6 mice for 35 days. Ad-shRNA particles targeting Cyp17A1 were generated using pAD_BLOCK_IT_DEST vectors (Invitrogen). The 2 shRNAs designed for the knockdown of Cyp17A1 had the following target sequences: C1: 5'-GCCTTTGCGGATAGTAGTAGC-3'; and C2: 5'-GCAGGCATAGAGACAACTAGC- $3^{\prime}$. For 15 days, $4 \times 10^{9} \mathrm{PFUs}$ per mouse were delivered into $d b / d b$ mice. All viruses were purified by the cesium chloride method, dialyzed in PBS containing 10\% glycerol, and administered to mice through tail vein injection.

ELISA analysis. For ELISA measurements of hepatic 17-OHP, freshly frozen liver tissues were homogenized in buffer containing 150 $\mathrm{mmol} / \mathrm{L} \mathrm{NaCl}, 10 \mathrm{mmol} / \mathrm{L}$ HEPES (pH 7.4), and 0.5\% Triton X-100 plus antiprotease cocktail (MilliporeSigma). 17-OHP concentrations in the liver and plasma were measured by commercial kits (catalog FRE2800, Labor Diagnostika Nord). Plasma insulin, ACTH, and corticosterone levels were measured using kits from Crystal Chem (catalog 90080), Phoenix Peptide (catalog FEK-001-21), and Labor Diagnostika Nord (catalog ARE-8100), respectively.

Ligand-binding analysis. FRET assays were performed using the LanthaScreen TR-FRET glucocorticoid receptor coactivator assay (A15899, Thermo-Fisher). Briefly, a GR ligand-binding domain that was tagged with glutathione-S-transferase (GST) and a fluoresceinlabeled coactivator peptide were incubated with Dex or 17-OHP for 20 minutes. Fluorescence was detected with a multireader with a background emission at $495 \mathrm{~nm}$ and binding signal at $520 \mathrm{~nm}$ after excitation at $340 \mathrm{~nm}$. We then generated a binding curve by plotting the emission ratio versus the log (ligand). The $y$ axis shows the ratio of fluorescence intensity at $520 \mathrm{~nm}$ (signal) over intensity at $495 \mathrm{~nm}$ (background). The $x$ axis represents the log scale of concentrations of 17-OHP or Dex in Figure 1I. To determine the $\mathrm{EC}_{50}$ value, we fitted the data using an equation for a sigmoidal dose response, as plotted by GraphPad Prism 6.0.

PTTs, GTTs, and ITTs. PTTs and GTTs were performed by i.p. injecting the mice with $1.5 \mathrm{mg} / \mathrm{kg}$ pyruvate (MilliporeSigma) or $2 \mathrm{mg} / \mathrm{kg}$ D-glucose (Sigma-Aldrich) after a 16-hour fast. For the ITTs, the mice were injected with regular human insulin (Eli Lily) at a dose of $0.75 \mathrm{U} / \mathrm{kg}$ body weight after a 6-hour fast. Blood glucose levels were then determined using a portable blood glucose meter (Lifescan, Johnson \& Johnson).

Cell culture. HepG2 and Hep1-6 cells were obtained from Cell Bank of Shanghai Institute of Biochemistry and Cell Biology. MPHs were isolated from the livers of C57BL/ 6 mice aged 10 weeks by collagenase perfusion and then purified by centrifugation. Freshly prepared MPHs were resuspended in attachment media (Science Cell) and seeded in 6-well plates at a final density of $5 \times 10^{5}$ cells per well. The media were replaced with DMEM (Gibco, Thermo Fisher Scientific) 12 hours after plating.

Plasmids, transfections, and luciferase reporter assays. HA-tagged full-length mouse AR, ER $\alpha, F X R, G R$, and LXR $\alpha$ were obtained by PCR amplification of the cDNA from mouse liver and cloned into pCMV vector with HindIII/BamHI restriction sites. The PCR primer sequences are listed in Supplemental Table 3. ERE-luciferase and FXR-luciferase plasmids, which harbored 3 ER and FXR responsible elements, respectively, were used in our previous studies $(69,70)$.
ARE-luciferase plasmid was provided by Jinke Cheng (Department of Biochemistry and Molecular Cell Biology, Shanghai Jiao Tong University School of Medicine, Shanghai, China) (71). LXRE-luciferase plasmid was provided by Youfei Guan (Advanced Institute for Medical Sciences, Dalian Medical University, Dalian, China) (72). For luciferase reporter assays, HepG2 cells were placed in 24-well plates and transfected with indicated reporter vectors (200 ng) and expression plasmids (50 ng) in duplicate wells using Lipofectamine 3000 (Thermo Fisher) according to the manufacturer's instructions. Total DNA used in each transfection was adjusted equally by adding appropriate amounts of empty vectors. pRL-TK-expressing renilla luciferase (Promega) was used to normalize the luciferase activity. Sixteen hours after transfection, cells were deprived of FBS for 12 hours and treated with various compounds or vehicle control (ethanol) for another 24 hours. Luciferase activities were measured using the Dual-Luciferase Reporter Assay System (Promega).

RNA isolation and real-time qPCR. Total RNAs from mouse livers or cultured MPHs were extracted using TRIzol reagents (Invitrogen). RNA purity and concentrations were measured using the NanoDrop ND-2000 spectrophotometer (Thermo Fisher Scientific). The mRNA were then reversed transcribed into cDNA using the Promega Reverse Transcription System. Oligo dT was used to prime cDNA synthesis. cDNA was stored at $-20^{\circ} \mathrm{C}$ before use. Real-time qPCR was performed using SYBR Green Premix Ex Taq on a Light Cycler 480 (Roche), and data were analyzed by the comparative CT method. PCR conditions included an initial holding period at $95^{\circ} \mathrm{C}$ for 5 minutes, followed by a 2-step PCR program consisting of $95^{\circ} \mathrm{C}$ for 5 seconds and $60^{\circ} \mathrm{C}$ for 30 seconds for 50 cycles. Relative abundance of mRNA was normalized to ribosomal protein 36B4. The primers for real-time qPCR are listed in Supplemental Table 4.

Western blots. To prepare total protein extracts, hepatic tissues and cells were lysed in RIPA buffer containing $50 \mathrm{mM}$ Tris- $\mathrm{HCl}$, $150 \mathrm{mM} \mathrm{NaCl}, 5 \mathrm{mM} \mathrm{MgCl}$, 2 mM EDTA, $1 \mathrm{mM} \mathrm{NaF,} \mathrm{1 \%} \mathrm{NP40,}$ and $0.1 \%$ sodium dodecyl sulfate. Cytoplasmic and nuclear protein fractions were prepared with the NE-PER Nuclear and Cytoplasmic Extraction Kit (Thermo Fisher Scientific) according to the manufacturer's instructions. For Western blots, equivalent amounts of protein samples were denatured in loading buffer and resolved by $10 \%$ to $12 \%$ sodium dodecyl sulfate polyacrylamide gel electrophoresis and transferred onto polyvinylidene fluoride membranes. Membranes were blocked in $5 \%$ nonfat milk for 1 hour before incubation with primary antibody overnight at $4^{\circ} \mathrm{C}$. Membranes were washed with PBS with Tween-20 (PBST) 5 times and incubated with secondary antibody for 1 hour. The signals of the proteins were then visualized by an electrochemiluminescence (ECL) system. The following primary antibodies were used: anti-Cyp17A1 at 1:2000 (catalog ab125022, Abcam), anti-GR at 1:2000 (catalog ab2768, Abcam), anti-phospho-Akt (S473) at 1:3000 (catalog 4060, Cell Signaling Technology), anti-total Akt at 1:2000 (catalog 4691, Cell Signaling Technology), anti-PR at 1:2000 (catalog ab2765, Abcam), anti-HSP90 at 1:5000 (catalog sc-69703, Santa Cruz), anti- $\alpha$-tubulin at 1:3000 (catalog ab7291, Abcam), anti-histone H3 antibody at 1:5000 (catalog 4499, Cell Signaling Technology), and antiGAPDH antibody at 1:8000 (catalog KC-5G5, Kangchen Technology). GAPDH and HSP90 were used as loading controls for total lysates. $\alpha$-Tubulin and histone $\mathrm{H} 3$ were used as loading controls for cytoplasmic and nuclear lysates, respectively. 
ChIP. A ChIP assay kit was used (MilliporeSigma). In brief, MPHs were treated with 17-OHP or vehicle control for 1 hour. Then cells were fixed in $1 \%$ formaldehyde (MilliporeSigma) for 10 minutes, washed, and harvested in SDS lysis buffer. DNA was sheared to fragments of 2001000 bp by several sonications. Lysates containing soluble chromatin were incubated and precipitated overnight with $5 \mu \mathrm{g}$ anti-GR antibody (catalog ab3579, Abcam) or rabbit IgG (catalog ab172730, Abcam). DNA protein-immune complexes were removed with protein $G$ agarose and then washed and eluted. Protein DNA crosslinks were reversed by treatment with proteinase $\mathrm{K}$ for 2 hours at $45^{\circ} \mathrm{C}$. The DNA was subsequently purified, diluted, and subjected to real-time qPCR. The mouse PEPCK and TAT gene promoter fragments containing the GRE motif were amplified using the following primers: PEPCK: 5'-TGCAGCCAGCAACATATGAA-3' (forward), 5'-TGATGCAAACTGCAGGCTCT-3' (reverse); and TAT: 5'-CGCAAACAACAGGAAGCCTAA-3' (forward), 5'-CATGACACCCAAAAGCCTCTC-3' (reverse). The promoter region of the GAPDH proximal promoter was set as the NC. Primers for the GAPDH promoter were 5'-CTATCCTGGGAACCATCA-3' (forward) and 5'-AAGCGTGTGGGCTCCGAA-3' (reverse).

All original microarray data were deposited in the NCBI's Gene Expression Omnibus database (GEO GSE129516).

Statistics. All statistical analysis was performed with SAS version 9.3 (SAS Institute). Data are presented as mean \pm SD or median (interquartile range). The human subjects were classified into different groups according to diabetic status or 4 quartiles according to plasma 17-OHP levels. A $\chi^{2}$ test was used to compare categorical variables between groups. ANOVA was performed using general linear models to test differences in study variables between groups or different quartiles of plasma 17-OHP levels. Data with an abnormal distribution were logarithmically transformed before analysis. Plasma 17-OHP levels and metabolic parameters were analyzed by Pearson's correlation. For animal and cellular experiments, a 2-tailed unpaired Student's $t$ test was performed to compare between 2 groups. One-way ANOVA followed by the Stu-
dent-Newman-Keuls test was used to compare more than 2 groups. $P$ values of less than 0.05 were considered statistically significant.

Study approval. The human study was approved by the Human Research Ethics Committees of Zhongshan Hospital, Fudan University, and Shanghai Jiao Tong University Affiliated Sixth People's Hospital. Written, informed consent was obtained from each subject. All animal protocols were reviewed and approved by the Animal Care Committee of Zhongshan Hospital, Fudan University.

\section{Author contributions}

YL and XL designed and directed the study. EW, BZ, JZ, LX, YQ, JJ, and XX performed animal and cellular experiments. YC, LZ, ZL, JY, RZ, HZ, and CH contributed to the human study. YC and HZ conducted the statistical analysis for the human study. DW and BL contributed to the study design and discussion. All authors reviewed the manuscript and provided final approval for submission.

\section{Acknowledgments}

This work was supported by the National Key Research and Development Program of China (2018YFA0800400 and 2016YFC1304801), the National Natural Science Foundation of China (81722013, 81870541, 81970736, and 81970748), the Outstanding Academic Leaders of Shanghai Health System (2017BR008), and Shanghai Municipal Education Commission-Gaofeng Clinical Medicine Grant Support (20152527).

Address correspondence to: Xiaoying Li, 180 Fenglin Road, Xuhui District, Shanghai 200032. Phone: 86.21.64041990.693403; Email:li.xiaoying@zs-hospital.sh.cn.Or to: Cheng Hu,600 Yishan Road, Xuhui District, Shanghai 200233. Phone: 86.21.24058247; Email: alfredhc@sjtu.edu.cn. Huijie Zhang, 1838 North Guangzhou Avenue, Guangzhou 510515. Phone: 86.20.61641635; Email: huijiezhang2005@126.com.
1. Preidis GA, Kim KH, Moore DD. Nutrient-sensing nuclear receptors PPAR $\alpha$ and FXR control liver energy balance. J Clin Invest. 2017;127(4):1193-1201.

2. Rui L. Energy metabolism in the liver. Compr Physiol. 2014;4(1):177-197.

3. Samuel VT, Shulman GI. The pathogenesis of insulin resistance: integrating signaling pathways and substrate flux. JClin Invest. 2016;126(1):12-22.

4. Könner AC, Brüning JC. Selective insulin and leptin resistance in metabolic disorders. Cell Metab. 2012;16(2):144-152.

5. Timmermans S, Souffriau J, Libert C. A general introduction to glucocorticoid biology. Front Immunol. 2019;10:1545.

6. Ferraù F, Korbonits M. Metabolic syndrome in Cushing's syndrome patients. Front Horm Res. 2018;49:85-103.

7. Rockall AG, et al. Hepatic steatosis in Cushing's syndrome: a radiological assessment using computed tomography. Eur JEndocrinol. 2003;149(6):543-548.

8. Howlett TA, Rees LH, Besser GM. Cushing's syndrome. Clin Endocrinol Metab. 1985;14(4):911-945.

9. Sakoda H, et al. Dexamethasone-induced insulin resistance in 3T3-L1 adipocytes is due to inhibition of glucose transport rather than insulin signal transduction. Diabetes. 2000;49(10):1700-1708.

10. Bernal-Mizrachi C, et al. Dexamethasone induction of hypertension and diabetes is PPAR-alpha dependent in LDL receptor-null mice. Nat Med. 2003;9(8):1069-1075.

11. John K, Marino JS, Sanchez ER, Hinds TD. The glucocorticoid receptor: cause of or cure for obesity? Am J Physiol Endocrinol Metab. 2016;310(4):E249-E257.

12. Vandewalle J, Luypaert A, De Bosscher K, Libert C. Therapeutic mechanisms of glucocorticoids. Trends Endocrinol Metab. 2018;29(1):42-54.

13. Bose SK, Hutson I, Harris CA. Hepatic glucocorticoid receptor plays a greater role than adipose GR in metabolic syndrome despite renal compensation. Endocrinology. 2016;157(12):4943-4960.

14. Hanson RW, Reshef L. Regulation of phosphoenolpyruvate carboxykinase (GTP) gene expression. Annu Rev Biochem. 1997;66:581-611.

15. van Schaftingen E, Gerin I. The glucose-6-phosphatase system. Biochem J. 2002;362(pt 3):513-532.

16. Cui A, et al. Dexamethasone-induced Krüppel-like factor 9 expression promotes hepatic gluconeogenesis and hyperglycemia. J Clin Invest. 2019;129(6):2266-2278.

17. Lee MW, et al. Regulation of hepatic gluconeogenesis by an ER-bound transcription factor,
CREBH. Cell Metab. 2010;11(4):331-339.

18. Liu Y, et al. Increased glucocorticoid receptor and 11 beta\}-hydroxysteroid dehydrogenase type 1 expression in hepatocytes may contribute to the phenotype of type 2 diabetes in $\mathrm{db} / \mathrm{db}$ mice. Diabetes. 2005;54(1):32-40.

19. Opherk C, et al. Inactivation of the glucocorticoid receptor in hepatocytes leads to fasting hypoglycemia and ameliorates hyperglycemia in streptozotocin-induced diabetes mellitus. Mol Endocrinol. 2004;18(6):1346-1353.

20. Lemke U, et al. The glucocorticoid receptor controls hepatic dyslipidemia through Hes1. Cell Metab. 2008;8(3):212-223.

21. Whirledge S, DeFranco DB. Glucocorticoid signaling in health and disease: insights from tissue-specific GR knockout mice. Endocrinology. 2018;159(1):46-64.

22. Abraham SB, Rubino D, Sinaii N, Ramsey S, Nieman LK. Cortisol, obesity, and the metabolic syndrome: a cross-sectional study of obese subjects and review of the literature. Obesity (Silver Spring). 2013;21(1):E105-E117.

23. Phillips DI, et al. Elevated plasma cortisol concentrations: a link between low birth weight and the insulin resistance syndrome? J Clin Endocrinol Metab. 1998;83(3):757-760. 
24. Ward AM, et al. Cortisol and the metabolic syndrome in South Asians. Clin Endocrinol (Oxf). 2003;58(4):500-505.

25. Stewart PM, Boulton A, Kumar S, Clark PM, Shackleton CH. Cortisol metabolism in human obesity: impaired cortisone->cortisol conversion in subjects with central adiposity. J Clin Endocrinol Metab. 1999;84(3):1022-1027.

26. Reynolds RM, et al. Elevated fasting plasma cortisol is associated with ischemic heart disease and its risk factors in people with type 2 diabetes: the Edinburgh type 2 diabetes study. JClin Endocrinol Metab. 2010;95(4):1602-1608.

27. Travison TG, O'Donnell AB, Araujo AB, Matsumoto AM, McKinlay JB. Cortisol levels and measures of body composition in middle-aged and older men. Clin Endocrinol (Oxf). 2007;67(1):71-77.

28. Janowski BA, Willy PJ, Devi TR, Falck JR, Mangelsdorf DJ. An oxysterol signalling pathway mediated by the nuclear receptor LXR alpha. Nature. 1996;383(6602):728-731.

29. Nelson ER. The significance of cholesterol and its metabolite, 27-hydroxycholesterol in breast cancer. Mol Cell Endocrinol. 2018;466:73-80.

30. Ma L, Nelson ER. Oxysterols and nuclear receptors. Mol Cell Endocrinol. 2019;484:42-51.

31. Patel R, et al. LXR $\beta$ is required for glucocorticoid-induced hyperglycemia and hepatosteatosis in mice. J Clin Invest. 2011;121(1):431-441.

32. Yang $X$, et al. Nuclear receptor expression links the circadian clock to metabolism. Cell. 2006;126(4):801-810.

33. Wang $\mathrm{X}$, et al. Hepatic estrogen receptor $\alpha$ improves hepatosteatosis through upregulation of small heterodimer partner. J Hepatol. 2015;63(1):183-190.

34. Schmitz G, Langmann T. Transcriptional regulatory networks in lipid metabolism control ABCA1 expression. Biochim Biophys Acta. 2005;1735(1):1-19.

35. Montagner A, et al. Liver PPAR $\alpha$ is crucial for whole-body fatty acid homeostasis and is protective against NAFLD. Gut. 2016;65(7):1202-1214.

36. Zhou J, et al. Hepatic fatty acid transporter Cd36 is a common target of LXR, PXR, and PPARga$\mathrm{mma}$ in promoting steatosis. Gastroenterology. 2008;134(2):556-567.

37. Aoki K, Terauchi Y. Effect of dehydroepiandrosterone (DHEA) on diabetes mellitus and obesity. Vitam Horm. 2018;108:355-365.

38. Veronese N, et al. Serum dehydroepiandrosterone sulfate and risk for type 2 diabetes in older men and women: the Pro.V.A Study. Can J Diabetes. 2016;40(2):158-163.

39. Brahimaj A, Muka T, Kavousi M, Laven JS, Dehghan A, Franco OH. Serum dehydroepiandrosterone levels are associated with lower risk of type 2 diabetes: the Rotterdam Study. Diabetologia. 2017;60(1):98-106.

40. Peng D, et al. Common variants in or near ZNRF1, COLEC12, SCYL1BP1 and API5 are associated with diabetic retinopathy in Chinese patients with type 2 diabetes. Diabetologia.
2015;58(6):1231-1238.

41. Wang T, et al. Causal association of overall obesity and abdominal obesity with type 2 diabetes: a Mendelian randomization analysis. Obesity (Silver Spring). 2018;26(5):934-942.

42. Yan J, et al. Impaired pancreatic beta cell compensatory function is the main cause of type 2 diabetes in individuals with high genetic risk: a 9 year prospective cohort study in the Chinese population. Diabetologia. 2016;59(7):1458-1462.

43. Zhang HJ, et al. Metabolic disorders in newly diagnosed young adult female patients with simple virilizing 21-hydroxylase deficiency. Endocrine. 2010;38(2):260-265.

44. Miller WL. Steroidogenic enzymes. Endocr Dev. 2008;13:1-18.

45. Miller WL, Auchus RJ, Geller DH. The regulation of 17,20 lyase activity. Steroids. 1997;62(1):133-142.

46. Anakk S, Watanabe M, Ochsner SA, McKenna NJ, Finegold MJ, Moore DD. Combined deletion of Fxr and Shp in mice induces Cyp17a1 and results in juvenile onset cholestasis. J Clin Invest. 2011;121(1):86-95.

47. Odermatt A, Klusonova P. 11 $\beta$-Hydroxysteroid dehydrogenase 1: Regeneration of active glucocorticoids is only part of the story. J Steroid Biochem Mol Biol. 2015;151:85-92.

48. Paterson JM, et al. Metabolic syndrome without obesity: Hepatic overexpression of 11beta-hydroxysteroid dehydrogenase type 1 in transgenic mice. Proc Natl Acad Sci U S A. 2004;101(18):7088-7093.

49. Heise T, et al. Safety, efficacy and weight effect of two 11 $\beta$-HSD1 inhibitors in metformin-treated patients with type 2 diabetes. Diabetes Obes Metab. 2014;16(11):1070-1077.

50. Li X, Wang J, Yang Q, Shao S. 11ß-Hydroxysteroid dehydrogenase type 1 in obese subjects with type 2 diabetes mellitus. Am J Med Sci. 2017;354(4):408-414.

51. de Bono JS, et al. Abiraterone and increased survival in metastatic prostate cancer. $N$ Engl J Med. 2011;364(21):1995-2005.

52. Ryan CJ, et al. Abiraterone in metastatic prostate cancer without previous chemotherapy. $N$ Engl J Med. 2013;368(2):138-148.

53. Li Z, et al. Conversion of abiraterone to D4A drives anti-tumour activity in prostate cancer. Nature. 2015;523(7560):347-351.

54. Xiao L, et al. Nuclear receptor LRH-1 functions to promote castration-resistant growth of prostate cancer via its promotion of intratumoral androgen biosynthesis. Cancer Res. 2018;78(9):2205-2218.

55. Mostaghel EA, et al. Resistance to CYP17A1 inhibition with abiraterone in castration-resistant prostate cancer: induction of steroidogenesis and androgen receptor splice variants. Clin Cancer Res. 2011;17(18):5913-5925.

56. Tucci M, et al. Abiraterone and prednisone therapy may cause severe hypoglycemia when administered to prostate cancer patients with type 2 diabetes receiving glucose-lowering agents.
Endocrine. 2019;64(3):724-726.

57. Yang LP. Abiraterone acetate: in metastatic castration-resistant prostate cancer. Drugs. 2011;71(15):2067-2077.

58. Del Re M, et al. The role of drug-drug interactions in prostate cancer treatment: Focus on abiraterone acetate/prednisone and enzalutamide. Cancer Treat Rev. 2017;55:71-82.

59. Meis PJ, et al. Prevention of recurrent preterm delivery by 17 alpha-hydroxyprogesterone caproate. N Engl JMed. 2003;348(24):2379-2385.

60. Deeks ED. $17 \alpha$-Hydroxyprogesterone caproate $\left(\right.$ Makena $\left.^{\mathrm{TM}}\right)$ : in the prevention of preterm birth. Paediatr Drugs. 2011;13(5):337-345.

61. Rebarber A, et al. Increased incidence of gestational diabetes in women receiving prophylactic 17alpha-hydroxyprogesterone caproate for prevention of recurrent preterm delivery. Diabetes Care. 2007;30(9):2277-2280.

62. Waters TP, Schultz BA, Mercer BM, Catalano PM. Effect of 17alpha-hydroxyprogesterone caproate on glucose intolerance in pregnancy. Obstet Gynecol. 2009;114(1):45-49.

63. Nelson DB, McIntire DD, McDonald J, Gard J, Turrichi P, Leveno KJ. 17-alpha Hydroxyprogesterone caproate did not reduce the rate of recurrent preterm birth in a prospective cohort study. Am JObstet Gynecol. 2017;216(6):600.e1-600.e9.

64. Pergialiotis V, Bellos I, Hatziagelaki E, Antsaklis A, Loutradis D, Daskalakis G. Progestogens for the prevention of preterm birth and risk of developing gestational diabetes mellitus: a meta-analysis. Am JObstet Gynecol. 2019;221(5):429-436.e5.

65. Eke AC, Sheffield J, Graham EM. 17 $\alpha$-Hydroxyprogesterone caproate and the risk of glucose intolerance in pregnancy: a systematic review and meta-analysis. Obstet Gynecol. 2019;133(3):468-475.

66. Dalle H, et al. Adipocyte glucocorticoid receptor deficiency promotes adipose tissue expandability and improves the metabolic profile under corticosterone exposure. Diabetes. 2019;68(2):305-317.

67. Mueller KM, et al. Adipocyte glucocorticoid receptor deficiency attenuates aging- and HFD-induced obesity and impairs the feeding-fasting transition. Diabetes. 2017;66(2):272-286.

68. Lu Y, et al. Periostin promotes liver steatosis and hypertriglyceridemia through downregulation of PPAR $\alpha$. J Clin Invest. 2014;124(8):3501-3513.

69. Li XY, et al. G protein-coupled receptor 48 upregulates estrogen receptor alpha expression via cAMP/PKA signaling in the male reproductive tract. Development. 2010;137(1):151-157.

70. Li J, et al. DAX1 suppresses FXR transactivity as a novel co-repressor. Biochem Biophys Res Commun. 2011;412(4):660-666.

71. Cheng J, Wang D, Wang Z, Yeh ET. SENP1 enhances androgen receptor-dependent transcription through desumoylation of histone deacetylase 1. Mol Cell Biol. 2004;24(13):6021-6028.

72. Wu J, et al. Liver X receptor-alpha mediates cholesterol efflux in glomerular mesangial cells. Am J Physiol Renal Physiol. 2004;287(5):F886-F895. 